\title{
XII. Auszüge.
}

1. B. Hasselbery (in Stockholm): Ueber das Vorkommen des Vanads in den skandinavischen Rntllarten (Bihang till Vet.-Akad. Handl. 1896, 22, Abtheil. I, Nr. 7). Nr. 3).

Derselbe: Zur chemischen Constitution des Rutils (Ebenda 1897, 23, I,

Verf. hat Rutile von 11 verschiedenen Fundorten spectroskopisch untersucht und dabei in sämmtlichen einen Gehalt an Vanad gefunden. Gerade die vanadreichsten von diesen Rutilen oder diejenigen von Käringbricka in Schweden, Langö und Loftushagen in Norwegen, Miask in Russland und Graves Mountain in Nordamerika zeigten sich zugleich chromhaltig. Von sonstigen Verunreinigungen enthalten diese Rutile nur Eisen. In dem zum Vergleich herangezogenen Anatas vom Binnenthal konnte dagegen weder Vanad noch Chrom nachgewiesen werden.

$$
\text { Ref.: H. Bäckström. }
$$

2. H. Weibnll (in Alnarp, Åkarp): Ueber Gedritschiefer vom südlichen Dalekarlien (Geol. Fören. Förh. 1896, 18, 377).

Unter den krystallinen Schiefern von Vester Silfberg in Dalekarlien findet sich ein Gedrilschiefer, charakterisirt durch das Vorkommen von langen Nadeln von Gedrit in Büscheln. Die optische Orientirung ist, wie gewöhnlich, $a=\mathfrak{a}$, $b=\mathfrak{b}, c=\mathfrak{c}$. Der Pleochroismus ist sehr ausgeprägt:

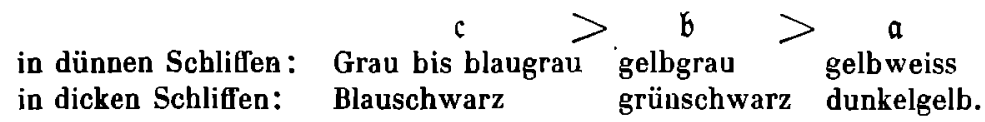

Eine von J. Petrén ausgeführte Analyse ergab:

\begin{tabular}{|c|c|c|c|}
\hline & & Molekularve & erhältniss \\
\hline $\mathrm{SiO}_{2}$ & 47,40 & 10 & \\
\hline $\mathrm{Al}_{2} \mathrm{O}_{3}$ & 13,68 & 1,68 & \\
\hline $\mathrm{FeO}$ & 23,38 & $4,11)$ & \\
\hline $\mathrm{MnO}$ & 2,36 & 0,42 & \\
\hline $\mathrm{MgO}$ & 7,32 & 2,32 & \\
\hline $\mathrm{Na}_{2} \mathrm{O}$ & 3,21 & 0,66 & 8,89 \\
\hline$K_{2} O$ & Spur & -1 & \\
\hline $\mathrm{H}_{2} \mathrm{O}$ & 1,97 & 1,38 & \\
\hline & $\overline{99,32}$ & & \\
\hline
\end{tabular}


Das Wasser wurde direcl gewogen. Ein Gehalt an Fluor wurde nachgewiesen, aber nicht quantitativ bestimmt. Spec. Gew. des Minerals $=3,243$.

Ref.: H. Bäckström.

3. M. Welbull (in Alnarp, Åkarp, Schweden): Ueber die Stellnng des Bliabergit im Mineralsysteme (Geol. Fören. Förh 1896, 18, 515).

H. Weiball und A. Upmark: Ueber den sog. Dicksbergit von Ransäter in Wermland (Ebenda S. 523).

Der "Bliabergit " Igelström's (s. diese Zeitschr. 27, 603) ist 0ttrelith (Chloritoid), wie der Ref. nach mikroskopischer Untersuchung gleich hervorhob (in einer Fussnote Geol. Fören. Förh. 1896, 18, 386). Zu demselben Resultate kam bald darnach nach chemischer Untersuchung Weibull. Auf eine Wiedergabe der an unreinem Material ausgeführten Analysen wird bier verzichtet.

Der ebenfalls von Igelström (Geol. Fören. Förh. 1896, 18, 231) aufgestellte "Dicksbergit " wurde von Weibull und Upmark als Rutil erkannt; sie fanden darin $98,13 \%, \mathrm{TiO}_{2}$.

Ref.: H. Bäckström.

4. H. Bäckström (in Stockholm): Ueber Manganandalusit von Vestanå (Ebenda 386).

In der Muscovitquarzilformation des nordöstlichen Schonens kommt, ausser Disthen, gewöhnlichem Andalusit, Ottrelith, Turmalin, bisweilen Fibrolith; auch ein grasgrünes Mineral vor, dessen nähere Untersuchung zeigte, dass es eine manganreiche Varietät von Andalusit war. Das Mineral ist unangreifbar durch Säuren und liess sich deshalb durch Flusssäure rein darstellen. Eine von Dr. H. Santess on ausgeführte Analyse ergab:

$$
\begin{array}{lrr}
\mathrm{SiO}_{2} & 36,72 & 0,6098 \\
\mathrm{Al}_{2} \mathrm{O}_{3} & \mathbf{5 6 , 9 9} & 1,1179 \\
\mathrm{Mn}_{2} \mathrm{O}_{3} & \mathbf{6 , 9 1} & \mathbf{0 , 0 8 7 8} \\
\mathrm{CaO} & \mathrm{Spur} & \\
\cline { 2 - 3 } & \mathbf{1 0 0 , 6 2} &
\end{array}
$$

Berechnet :$$
36,23
$$$$
56,87
$$$$
6,90
$$$$
\overline{100,00}
$$

Nebenbei sind die nach der Andalusitformel $(A l, \stackrel{\mathrm{MI}}{\mathrm{MI}})_{2} \mathrm{SiO}_{5}$ berechneten Werthe aufgeführt. Der höchste bis jetzt in Andalusit angetroffene Manganoxydgehalt war $0,83 \%$ (in einer rothen Varietät von Lisens in Tirol), daher dieser Manganandalusit als eine gut charakterisirte Varietät zu betrachten ist.

Der Manganandalusit unterscheidet sich in Bezug auf Unangreifbarkeit durch Säuren, Härte, Spaltbarkeit, sowie Lichtbrechung, Doppelbrechung und optischer Orientirung nicht wesentlich von dem gewöhnlichen Andalusit, wohl aber durch die grasgrüne Farbe und den kräftigen Pleochroismus, welcher auch in Dünnschliffen immer stark ausgeprägt ist. Es $\operatorname{sind} a=\mathfrak{c}$ und $b=\mathfrak{b}$ blaugrün mit einem Stich ins grasgrüne, $c=\mathfrak{a}$ wird am stärksten absorbirt und zeigt eine intensive, rein gelbe Farbe.

In einigen Gesteinen zeigt sich der Manganandalusit randlich zu Kaolin oder feinblättrigem Muscovit umgewandelt, und dann sind Doppelbrechung und Farbe schwächer, als wenn das Mineral ganz frisch ist. In einem Falle wurden manganandalusitartige Kerne in gewöhnlichem Andalusiṭ angetroffen.

Ref.: H. Bäckström. 
5. J. J. Sederholm (in Helsingfors) : Ueber Maltesit, eine chistolithartige Andalusitvarietät vom östlichen Finnland (Ebenda 390 ).

In den krystallinen Schiefern der Gegend nördlich vom Ladogasee wurden in den Kirchspielen Ruskeala und Impilaks grosse Andalusitknauern von eigenthümlicher Structur angelroffen. Sie sind wie die Chiastolithe aus abwechselnden Partien reiner und unreiner Substanz aufgebaut, aber hier besteht der Krystall aus einem Kreuz von reiner Substanz, dessen - wie bei einem Malteserkreuz nach innen schmäler werdende Theile gegen die vier Prismenflächen gerichlet sind, und dieses Kreuz wird durch eine mit den Schiefermineralien vollgeptropfte Andalusitsubstanz umgeben. Wie beim Chiastolith entsprechen also auch hier die reinen Partien gegen die Prismenflächen gerichteten Anwachskegeln, obwohl das Aussehen ein verschiedenes ist.

Ref.: H. Bäckström.

\section{K. Winge (in Stockholm): Ueber Calcit von Nordmarken (Ebenda 527 ).}

An dem bekannten Mineralfundorte Nordmarken in Wermland kommen auch zum Theil schöne und grosse Calcitkrystalle von wechselnder Ausbildung vor. Sie werden getheilt in:

I. Krystalle mit rhomboëdrischem Habitus; Combinationen: 1) $\{10 T 1\}$. 2) $\{10 \bar{T}\},\{7.4 . \bar{T} T .3\},\{0001\}$.

II. Krystalle mit skalenoëdrischem Habitus; Combinationen: 1 a) $\{21 \overline{3} 1\}$, allein oder mit $\{10 \overline{1} 0\},\{40 \overline{4} 1\},\{70 \overline{7} 1\},\{31 \overline{4} 5\}$; ferner $1 \mathrm{~b})\{21 \overline{3} 1\},\{10 \overline{1}\}$. 2a) $\{53 \overline{8} 2\},\{40 \overline{4} 1\},\{10 \bar{T} 0\},\{41 \overline{5} 6\}$. 2 b) $\{53 \overline{8} 2\},\{10 \bar{T} 0\},\{40 \overline{4} 1\},\{41 \overline{5} 6\}$, $\{41 \overline{5} 3\},\{10 \bar{T} 1\} .3)\{7.4 . \overline{1} \overline{1} .3\},\{40 \overline{4} 1\},\{10 \bar{T} 0\},\{34 \overline{7} 1\},\{21 \overline{3} 4\},\{0001\}$. 4) $\{19.13 . \overline{3} \overline{2} .6\},\{42 \overline{6} 1\},\{21 \overline{3} 1\},\{40 \overline{4} 1\},\{10 \overline{1} 1\}$.

III. Krystalle mit prismatischem Habitus; Combinalionen: 1) $\{10 \overline{1} 0\}$, $\{7.4 . \overline{1} \bar{T} .3\},\{10 \bar{T} 1\} .2)\{10 \bar{T} 0\},\{21 \overline{3} 4\},\{0001\} .3)\{10 \bar{T} 0\},\{43 \overline{7} 1\} .4)\{10 \bar{T} 0\}$, $\{53 \overline{8} 2\},\{31 \overline{4} 5\},\{40 \overline{4} 1\},\{10 \overline{1} 1\}$. 5) $\{10 \overline{1} 0\},\{11 \overline{2} 0\},\{21 \overline{3} 1\},\{44 \overline{8} 1\},\{11 \overline{2} 3\}$.

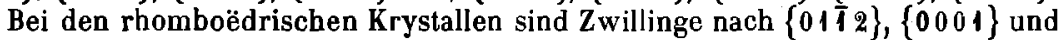
$\{10 T 1\}$ beobachtet, erstere häufig, lelztere selten; bei den skalenoëdrischen Krystallen sind Zwillinge nach $\{0001\}$ häufig, nach $\left\{01 \overline{1}_{2}\right\}$ dagegen selten. Bezüglich des relativen Alters der verschiedenen Typen liess sich bestimmen, dass die skalenoëdrischen Typen II. $1 \mathrm{~b}$ und II. $2 \mathrm{~b}$ jünger als die rhomboëdrischen I. 1 sind, und dass die prismatischen Krystalle III. 3 jünger als die skalenoëdrischen II. $1 \mathrm{~b}$ sind. Von den begleitenden Mineralien sind Diopsid, Hornblende, Chloril, Epidot und Magnetit älter, der Schwefelkies dagegen gleichaltrig mit dem Calcil.

Ref.: H. Bäckström.

7. A. Abt (in Klausenburg): Ueber die im Eisenerzlager von Moravioza vorkommenden natürlichen Hagnete (Math. és term. tud. Ertesitö 1896, 14, $33-41$, ung.).

Verf. fand, dass unter den magnetischen Körpern der Magnetit den grössten specifischen Magnetismus besitzt, wie dies schon aus den Untersuchungen von Holz bekannt war, sogar in einem Maasse, welches den des Stahles noch beträchtlich übertreffen kann. Ein Pyrrhotin, dessen Eisengehalt 57,58\% betragen hat, besass einen um 3,56 kleineren remanenten Magnetismus als der Magnetit; im Verhältniss zum Stahl 0,66. Der Hämatit von Moravicza besitzt einen specifischen Magnetismus von 0,214 im Verhältniss zum Stahl. Verf. hat auch die Polarität der Magnetitmassen zu Moravicza in situ untersucht und gefunden, dass dieselbe derart wechselt, dass nach diesen ersten Untersuchungen 
noch kein sicherer Schluss zu ziehen isl. Er stellte ferner fest, dass unter den Magnetitslücken manchmal derartige natürliche Magnete zu treflen sind, die eine beträchtliche Quantität Magnetismus besitzen; so wechselte unter zehn untersuchten Magnetiten der relative specifische Magnetismus von 0,0144 bis 0,0380. Aber auch unter den Pyrrhotinstücken kommen beträchtliche natürliche Magnete vor. Auch die Tragkraft des Magnetits hat Verfasser untersucht und fand nach geeigneten Vorrichtungen, dass ein $1008 \mathrm{~g}$ schwerer Magnetit den Anker erst bei einer Belastung von $2730 \mathrm{~g}$ fallen liess. Nach einigen, den Eisenerzbergbau zu Vaskö erläuternden Daten fand Verf. schliesslich, dass, wenn man das magnetische Moment der aus verschieden eisenhaltigen Magnetiten geschnitlenen und bis zur Sältigung magnetisirten Prismen mit dem in ihnen enthaltenen gesammten Eisengehalte vergleicht, eine annähernd constante Verhältn isszahl resultirt.

Ref.: A. Schmidt.

8. G. Melczer (in Budapesi): Daten zar krystallographischen Kenntniss des Calcites rom kleinen Schwabenberge bei Budapest (Földtani Közlöny 1896, 26, $10-25$ ung., 79-94 deutsch).

Auf Grund der Untersuchung einer grösseren Suite des oben angegebenen Vorkommens giebt Verf. eine detaillirte Studie der neueren Vorkommnisse. Unter den nicht häufigen $Z$ willingskrystallen kann man nach der Basis gebildete einfache Zwillinge, Wiederbolungsdrillinge und -Vierlinge und Penelrationszwillinge unterscheiden. Die sämmtlichen, bis jetzt am Calcil vom kleinen Schwabenberge bekannten Formen sind nach Verf. die folgenden: $m\left\{10 T_{0}\right\} \infty R, r\left\{10 \bar{T}_{1}\right\} R$, $l\{30 \overline{3} 1\} 3 R, M\{40 \overline{4} 1\} 4 R, \quad \delta\{90 \overline{9} 1\} 9 R, \varrho\{16.0 . \overline{1} \overline{1} 1\} 16 R, e\{01 \overline{1} 2\}-\frac{1}{2} R$, $f\{02 \overline{2} 1\}-2 R, * g\{0.16 . \overline{1} \overline{6} .5\}-\frac{16}{5} R, \pi\{1 \overline{2} 3\} \frac{2}{3} P 2, v\{21 \overline{3} 1\} R 3, * m\{52 \overline{7} 1\}$ $\left.3 R \frac{7}{3},{ }^{*} \mathfrak{n}\{63.28 . \overline{9} \overline{1} .11\}\right\}_{15}^{3} R L_{5}^{3}, \quad t\{2134\} 1 R 3, \quad g:\{52 \overline{7} 9\} \frac{1}{3} R \frac{7}{3}, E\{41 \overline{5} 6\} \frac{1}{2} R \frac{5}{3}$,

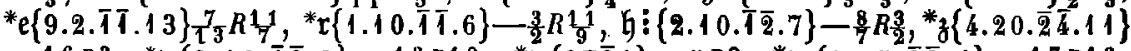

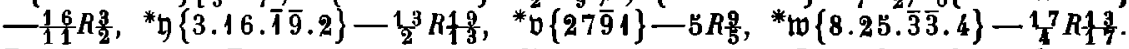
Zusammen 23 Formen, worunter die 9 mil einem Sternchen bezeichneten neu sind. Aus den Messungen des Verfassers können die nachstehenden erwähnt werden :

\begin{tabular}{|c|c|c|c|c|c|}
\hline$(52 \overline{7} 1):(7 \overline{2} \overline{5} 1)$ & $\begin{array}{r}\text { Beobs } \\
=31\end{array}$ & $\begin{array}{l}\text { chtet: } \\
23^{\prime}\end{array}$ & \multicolumn{3}{|c|}{$\begin{array}{l}\text { Berechnel: } \\
31^{0} 46^{\prime} 38^{\prime \prime}\end{array}$} \\
\hline$(52 \overline{7} 1):(21 \overline{3} 1)$ & $=12$ & 34 & 12 & 5 & 45 \\
\hline$(52 \overline{7} 1):(12 \overline{3} \overline{1})$ & $=38$ & 52 & 38 & 47 & 17 \\
\hline $3.28 . \overline{9} \overline{1}$ & $\begin{array}{r}=33 \\
36\end{array}$ & $\left.\begin{array}{l}24 \\
26\end{array}\right\}$ & 34 & 37 & 16 \\
\hline$(63.28 . \overline{9} \bar{T} .11):(21 \overline{3} 1)$ & $=13$ & 7 & 13 & 11 & 42 \\
\hline $3.28 . \overline{9} T .11):(12 \overline{3} \bar{T})$ & $=36$ & 45 & 36 & 52 & 44 \\
\hline$(16.0 . \overline{1} \overline{6} .1):\left(10 T_{0}\right)$ & $=3$ & 46 & 3 & 37 & 31 \\
\hline$(0.16 . \overline{1} \overline{6} .5):(02 \overline{2} 1)$ & $=9$ & 12 & 9 & 18 & 3 \\
\hline is 7 & 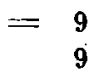 & $\begin{array}{r}6 \\
29\end{array}$ & 9 & 30 & 56 \\
\hline 1):(21 & $=30$ & $\mathbf{B 7}$ & 31 & 0 & 57 \\
\hline 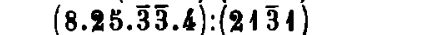 & $=29$ & 26 & 29 & 37 & 33 \\
\hline$(4.20 . \overline{2}$ & $=29$ & 15 & 29 & 41 & 34 \\
\hline$(3.16$ & $=22$ & 6 & 21 & 52 & 12 \\
\hline$(1.10$ & $=4 \mathbf{b}$ & 32 & 45 & 44 & 40 \\
\hline
\end{tabular}

Zur Grundlage der Rechnung wurde der Werth von: $(0001):(10 \overline{1} 1)=$ 
$44^{0} 36^{\prime} 3 h^{\prime \prime}$ angenommen. Wegen des näheren Delails muss aul das Original hingewiesen werden, welches auch mit zwei Taleln Kryslallzeichnungen versehen isl.

lief. : A. Schmidt.

9. J. v. Szádeczky (in Klausenburg): Cölestin vom Gebel El-Ahmar in Egypten (Földtani Közlöny 1896, 26, 113-116 ung., 161-165 deutsch).

Bei einer Excursion (1896) fand Verfasser in einem verlassenen Steinbruche des Gebel El-Ahmar (Berg in der Umgegend von Kairo) auf eocänem Kalkstein eine Cölestinkrystalldruse. Die Krystalle sind farblos, wasserklar, und erreichen eine Grösse von 1,4 cm; dieselbe besitzen die einfache Combination der Formen: $c\{001\}, m\{110\}, o\{011\}, d\{102\}$ und $l\{104\}$. Habilus ausgesprochen prismatisch nach dem Spaltungsprisma $m\{110\}$, wobei an deu kleineren Krystallen auch noch o\{0 11$\}$ vorwaltet, während bei den grösseren die Spaltungsendfläche $c\left\{\begin{array}{lll}0 & 0\end{array}\right\}$ gross ausgebildet ist. Die Messungen des Verf. gaben die nachstehenden Werthe:

$$
\begin{aligned}
& \text { Beobachtet: Berechnet: }
\end{aligned}
$$

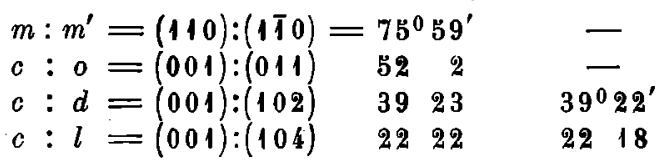

Spectroskopisch wurde keine Spur von Baryum und Calcium in diesen Krystallen gefunden. Die zwei anderen Fundstellen des Cölestins in Egyplen sind der Mokkatam (etwa $5 \mathrm{~km}$ südw. von El-Ahmar) und der neben liegende Wadi el-Tih, auf der südlichen Seite von Kairo.

Ref.: A. Schmidt.

10. G. Melezer (in Budapest) : Baryt ron Dobsina (Ebenda $321-324$ ung., $367-360$ deutsch).

Baryt in ausgebildeten Krystallen wurde zu Dobsina erst im Jahre 1890 auf Siderit aufgewachsen in den Eisenspathgruben "Massörter " gefunden. Die makrodiagonal gestreckten domatischen Krystalle sind 0,5-1,5 cm lang, etwas gelblich oder weisslich, mehr oder weniger durchsichtig. Die Formen dieser Krystalle sind: $a\{100\}, b\{010\}, c\{001\}$ Spaltung, $m\{110\}$ Spaltung, $\lambda\{210\}$, $\eta\{320\}, \chi\{130\}, u\{101\}, d\{102\}, l\{104\}, o\{011\}, z\{111\}, r\{112\}, f\{113\}$, $q\{114\}, v\{115\}, *^{*}\{772\}$ und $y\{122\}$, zusammen 18 Formen, darunter $p\{772\}$ eine neue Form. Die grösst-ausgebildeten Flächen sind gewöhnlich $d\left\{\begin{array}{ll}1 & 02\end{array}\right\}$, $m\{110\}$ und $c\{001\}$, welche auch den Habitus bestimmen. Aus den Messungen des Verf. mögen die folgenden aufgeführt werden:

Beobachtet:

$$
\begin{aligned}
& m: a=(110):(100)=39^{0} 10^{\prime} \\
& c: d=(001):(102) \quad 3852 \\
& m: z=(110):(111) \quad 2539 \\
& m: \mathfrak{p}=(110):(772) \quad 745 \\
& o: y=(011):(122) \quad 2558
\end{aligned}
$$

$\begin{array}{rr}\text { Berechnet: } \\ 39^{0} & 10^{\prime} \\ 38 & 52 \\ 25 & 42 \\ 7 & 50 \\ 26 & 2\end{array}$

Die berechneten Werthe sind aus Miller's Grundwerthen abgeleitet. Spectroskopisch konnte in diesen Krystallen von den Basen blos $B a$ nachgewiesen werden. Auf einer $\| \mathrm{zu}(\mathbf{1 0 0})$ geschliffenen Platte wurde gefunden im $\mathrm{Na}$-Lichte : 
$2 E_{a}=66^{\circ} 17^{\prime}, 2 H_{a}=44^{0} 34^{\prime} ;$ dieselben Winkel waren, an einem Barylkrystall von Budapest (kl. Schwabenberg) gemessen : $2 E_{a}=67^{\circ} 27^{\prime}$ und $2 H_{a}=44^{\circ} 37^{\prime}$.

Ref.: A. Schmidt.

11. G. Nyiredy (in Klausenburg) : Analysen einiger Mineralien (Ërtesitö az erd. Muzeurn egyl. orv.-lerm.-tud. szakosztályából. 1896, 21, 123-128 ung., 29-31 deutsch).

1) Magnetil von Vaskb̋. Derb, specif. Gew. 4,696, enthält im Mittel $\mathrm{SiO}_{2}$ 5,72, $\mathrm{Fe}$ 68, 10, O 25,93, Summe: 93,75\%.2) Pyrrhotin. a) Von Borév 1), b) von Ó-Radna, c) von Oravicza:

\begin{tabular}{|c|c|c|c|}
\hline \multirow{4}{*}{$\begin{array}{l}\mathrm{SiO}_{2} \\
\mathrm{Fe} \\
\mathrm{S}\end{array}$} & a. & b. & c. \\
\hline & 4,42 & 4,92 & 7,25 \\
\hline & 57,68 & 57,30 & 55,92 \\
\hline & 37,66 & 37,42 & 36,48 \\
\hline & 99,76 & 99,64 & 99,65 \\
\hline
\end{tabular}

12. A. Abt (in Klausenburg): Leitungswiderstand and speciflsche Wärme einiger Eisenoxyde und Eisensulflde (Ebenda 210-216 ung., 42-48 deutsch).

In dieser Arbeit physikalischer Natur werden die Leitungswiderstände von Nickelerzen aus Dobsina, Pyrrhotin von Alsó-Jára, Chalkopyrit von Oláhlápos, Pyrit von Felsöbánya und Dognácska, Magnetit von Moravicza, Hämatit von Kakukhegy (Hargita) und Moravicza, Siderit von Dobsina bestimmt. Die grossen Differenzen in dem Leitungswiderstande einiger der untersuchten Erze betrachtet Verf. als durch Verunreinigungen hervorgerufene Erscheinungen. Die specifischen Wärmen hat Herr Jobann Jepure bestimmt; er fand im Mittel die folgenden Calorien: Pyrrhotin von Alsó Jára: 0,15391; Magnelit von Moravicza: 0,16582; Hämatil von Dognácska: 0,17486; Nickelerz von Dobsina: 0,10412.

$$
\text { Ref.: A. Schmidt. }
$$

13. L. Brugnatelli (in Pavia): Krystallographisehe und optische Untersuchung des Oxydimethylnaphtols (1.4.2.3) $\mathrm{C}_{12} \mathrm{H}_{12} \mathrm{O}_{2}$ (Gazzella chim. ital. 1896, 1, 22). Dargestellt von Cannizzaro und Andreocci. Schmelzpunki 10401050. Krystalle aus Essigäther. Triklin.

$$
\alpha=\begin{gathered}
a: b: c=0,8797: 1: 0,7877 . \\
00^{\circ} 18^{\prime}, \quad \beta=98^{\circ} 22^{\prime}, \quad \gamma=54^{0} 31^{\prime} .
\end{gathered}
$$

Beobachlete Formen: $\{100\},\{001\},\{010\},\{110\},\{011\},\{11 \overline{1}\}$.

\begin{tabular}{|c|c|c|}
\hline \multirow{2}{*}{\multicolumn{2}{|c|}{$\begin{array}{c}\text { Winkel: } \\
\left(\begin{array}{lll}1 & 0 & 0\end{array}\right):\left(\begin{array}{lll}0 & 0 & \text { Beobachtet }\end{array}\right)={ }^{*} 79^{0} 56^{\prime}\end{array}$}} & Berechn \\
\hline & & \\
\hline$(100):(0 \bar{T} 0)$ & $=*_{54} \quad 8$ & - \\
\hline$(001):(0 \bar{T} 0)$ & *84 22 & - \\
\hline$(0 \bar{\top} 0):(1 \backslash 1)$ & *71 36 & - \\
\hline$(\bar{\top} 00):(\bar{\top} 11)$ & *73 46 & - \\
\hline
\end{tabular}

Gelbliche, durchsichtige Krystalle. Theils tafelig entweder nach $\{100\}$ oder nach $\{001\}$, theils prismatisch nach [001].

1) Siehe diese Zeitschr. 27, 101. 


\begin{tabular}{|c|c|c|}
\hline $\begin{array}{c}\text { Winkcl : } \\
(100):(110)\end{array}$ & $\begin{array}{l}\text { Beobachlel: } \\
=55^{0} 12^{\prime}\end{array}$ & $\begin{array}{c}\text { Berechnel } \\
55^{0} 13^{\prime}\end{array}$ \\
\hline$\left(\begin{array}{lll}0 & 0 & 1\end{array}\right):(110)$ & $\begin{array}{ll}83 & 52\end{array}$ & 842 \\
\hline$\left(\begin{array}{lll}0 & 0 & 1\end{array}\right):\left(\begin{array}{lll}0 & 1 & 1\end{array}\right)$ & $46 \quad 34$ & 4626 \\
\hline 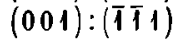 & 467 & $46 \quad 10$ \\
\hline$\left(0 \begin{array}{lll}0 & 0\end{array}\right):(011)$ & 4910 & $49 \quad 12$ \\
\hline$(\overline{1} 00):\left(\begin{array}{lll}0 & 1 & 1\end{array}\right)$ & $72 \quad 51$ & 7256 \\
\hline$(110):\left(11 T^{\prime}\right)$ & 4943 & 4948 \\
\hline
\end{tabular}

Vollkommene Spaltbarkeit nach $\{001\}$. Glasglanz. Durch die Spaltplalten trilt eine der optischen Axen und an der Grenze des Gesichtsfeldes die erste Mittellinie aus. Auf $\left\{\begin{array}{ll}0 & 0\end{array}\right\}$ bildet eine Auslöschungsrichtung gegen $+c$ und nach $+b$ einen Winkel von $22^{0}$ ca. (weisses Licht). Dispersion der optischen Axen sehr stark, $\varrho<v$. Doppelbrechung ausserordentlich stark und negativ.

Ref.: G. Bartalini.

14. G. B. Negri (in Genua): Krystallographische Untersuchnng einiger organischer Verbindungen (Ebenda 1, 68; 2, 186).

1. Paramidobenzoësäure.

Krystallsystem: Monoklin.

$$
a: b: c=1,4403: 1: 0,7312 ; \beta=100^{\circ} 10^{\circ} \text {. }
$$

Beobachtete Formen: $\{101\},\left\{\overline{1}_{01}\right\},\{110\},\{011\}$. Kleine, gut spiegeinde nach [ 100$]$ verlängerte Kryslalle. $\left\{\begin{array}{lll}1 & 0 & 1\end{array}\right\}$ öfters mil ausgehöhlten Flächen. $\left\{\begin{array}{lll}0 & 1\end{array}\right\}$ schmal und selten.

\begin{tabular}{|c|c|c|}
\hline $\begin{array}{c}\text { Winkel: } \\
(101):(110)\end{array}$ & $\begin{array}{l}\text { Beobachtet: } \\
=70^{\circ} 47^{\prime}\end{array}$ & $\begin{array}{l}\text { Berechnet } \\
70^{0} 47 \frac{1}{2}^{\prime}\end{array}$ \\
\hline$(101):(T 01)$ & 5323 & 5324 \\
\hline$(\overline{1} T 0):\left(\begin{array}{lll}1 & 01\end{array}\right)$ & 7924 & $7924 \frac{1}{2}$ \\
\hline$(110):(\overline{1} 10)$ & 7021 & 7024 \\
\hline$(110):(011)$ & 5555 & 5557 \\
\hline$(011):(\overline{1} 01)$ & 4433 & $44 \quad 39$ \\
\hline$(\overline{1} 10):(011)$ & 6655 & 6645 \\
\hline$(011):(101)$ & $42 \quad 17$ & 4228 \\
\hline
\end{tabular}

Nimmt man $\{101\},\left\{T_{01}\right\},\{110\},\{011\}$ als $\left\{T_{0} 3\right\},\{101\},\{013\},\{\overline{3} 23\}$, so erhält man die Constanten

$$
a: b: c=1,0968: 1: 4,2661 ; \beta=85^{0} 30 \frac{1}{2}^{\prime},
$$

welche ähnlich denen der Benzoësäure

$$
a: b: c=1,0511: 1: 4,2081 ; \beta=82^{\circ} 55^{\prime}
$$

sind, und deren berechnete Winkel ebenso sehr ähnlich sind. Nach dem Verf. besteht also "geometrische Isomorphie ( zwischen den beiden Subslanzen. Die einzige beiden gemeinsame Form ist $\left\{\begin{array}{lll}1 & 0 & 1\end{array}\right\}$.

\section{Pyroschleimsäure.}

Viele Versuche wurden gemacht, um für Messungen geeignete Krystalle zu erhalten, aber vergeblich; stets sind nur Flächen der Zone [001] vorhanden, nämlich $\{010\},\{120\},\{210\}$. Durch Spaltung erhielt man auch $\{011\}$. Die Anwesenheit dieser Spaltbarkeit und die schiefen Auslöschungsrichtungen auf den Flächen der Zone [ $\left[\begin{array}{lll}0 & 1\end{array}\right]$ zeigen, dass wahrscheinlich die Krystalle monoklin sind. In diesem Falle wären die Constanten 


$$
\begin{aligned}
& a: b: c=0,9556: 1: 0,5259 ; \beta \cdots 66^{0} 16^{\prime} .
\end{aligned}
$$

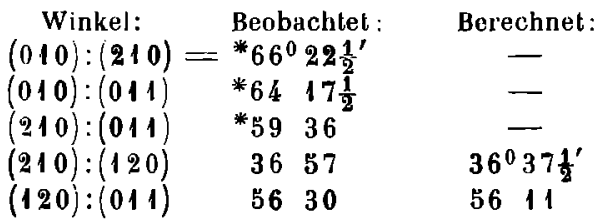

Das Verhältniss $a: b$ ist ähnlich bei Pyroschleimsäure, Benzoësäure und Carbopyrrholsäure; und $c: b$ der Pyroschleimsäure ist $\frac{1}{8}$ des Werthes von $c: b$ bei der Benzoësäure. Ausserdem ist bei den drei Substanzen ein Winkel der Zone $\left[\begin{array}{lll}0 & 1 & 0\end{array}\right]$ sehr ähnlich.

$$
\begin{aligned}
& \text { Pyroschleimsäure } \quad(100):(001)=66^{\circ} 16^{\prime} \\
& \text { Carbopyrrholsäure } \quad(100):(001) \quad 6710 \text { (Brezina) } \\
& \text { Benzoësäure } \quad(101):(001) \quad 6924
\end{aligned}
$$

3. Brenzcatechin.

Krystallsystem: Monoklin.

$$
a: b: c=1,6133: 1: ? ; \quad \beta=86^{0} 45^{\prime} \text {. }
$$

Beobachtete Formen: $\{100\},\{001\},\{110\}$. Nach $\{100\}$ tafelige Krystalle. Im Allgemeinen unvollkommene, gerundete, schlecht spiegelnde Flächen.

$$
\begin{aligned}
& \text { Winkel: Beobachlet: Berechnet: }
\end{aligned}
$$

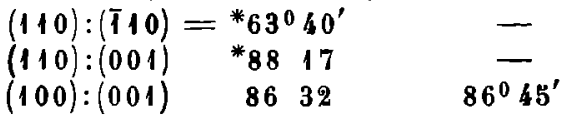

Spaltbarkeit vollkommen nach $\{100\}$; auf $(100)$ gerade Auslöschung.

Die folgenden Körper sind dargestellt von G. Pellizzari.

4. Amidophenylguanidinnitrat $\mathrm{C}_{7} \mathrm{H}_{10} \mathrm{~N}_{4} . \mathrm{NO}_{3} \mathrm{H}$.

\begin{tabular}{|c|c|c|c|}
\hline Winkel : & Beobachtet: & $\begin{array}{l}\text { Zahl der } \\
\text { Kanten: }\end{array}$ & Berechnet: \\
\hline$\left(\begin{array}{lll}1 & 0 & 1\end{array}\right):\left(\begin{array}{lll}1 & 0 & 1\end{array}\right)$ & $={ }^{*} 53^{0} 41^{\prime}$ & 6 & - \\
\hline$(110):(010)$ & ${ }^{*} 63 \quad 12$ & 8 & - \\
\hline$(\overline{1} 10):(\bar{T} 01)$ & ${ }^{*} 69 \quad 10$ & 6 & - \\
\hline$(110):(101)$ & $63 \quad 39 \frac{1}{2}$ & 2 & $63^{0} 19^{\prime}$ \\
\hline$(010):(\overline{1} 41)$ & 4656 & b & $46 \quad 43$ \\
\hline$(\overline{1} 41):(\bar{T} 01)$ & 4258 & 3 & 4317 \\
\hline$(T 41):(T 10)$ & 554 & 3 & 5523 \\
\hline$(341):(110)$ & 2812 & 2 & 2820 \\
\hline$(341):(010)$ & 6140 & 1 & 6126 \\
\hline
\end{tabular}

Schmelzpunkt $143^{\circ}$. Krystalle aus Wasser. Monoklin.

$$
a: b: c=0,5065: 1: 0,2567 ; \beta=85^{0} 47^{\prime} \text {. }
$$

Beobachtete Formen: $\{101\},\{10 T\},\{010\},\{110\},\{14 T\},\{341\}$. Farblose, prismatische, nach $[010]$ verlängerte Krystalle. Vorherrschend $\{101\},\{100\}$, $\left\{\begin{array}{lll}0 & 1 & 0\end{array}\right\}$. Messbare Krystalle sind selten.

Auf den Flächen der Zone [010] parallele Auslöschung. 
5. Benzylidenparatolylguanidinnilrat $\mathrm{C}_{15} \mathrm{H}_{16} \mathrm{~N}_{4}, \mathrm{NO}_{3} \mathrm{H}$.

Schmelzpunkt $214^{0}$. Krystalle aus Wasser. Monoklin.

$$
a: b: c=1,1659: 1: 0,8851 ; \beta=62^{0} 24^{\prime} \text {. }
$$

Beobachlete Formen: $\{110\},\left\{\overline{1}_{11}\right\},\left\{\overline{2}_{2} 3\right\}$. Wachsgelbe, gut spiegelnde Krystalle. Vorherrschend $\{110\}$.

\begin{tabular}{|c|c|c|c|}
\hline Winkel: & Beobachtet: & $\begin{array}{l}\text { Zahl der } \\
\text { Kanten : }\end{array}$ & Berechnet \\
\hline 110$):(1 \overline{1} 0$ & $={ }^{*} 91^{0} 52^{\prime}$ & 10 & - \\
\hline$\overline{1} 10):(\bar{T} 11)$ & ${ }^{*} 50 \quad 54$ & 6 & - \\
\hline$\overline{1} 11):(\overline{1} 11)$ & ${ }^{*} 80 \quad 2$ & 4 & 一 \\
\hline 110$):(\overline{1} 11)$ & 7311 & 4 & $72^{\circ} B 6^{\prime}$ \\
\hline 23$):(\bar{T} 11)$ & 1530 & 6 & 1555 \\
\hline 223$):(110)$ & 7025 & 3 & $70 \quad 22$ \\
\hline
\end{tabular}

Starker Pleochroïsmus durch $\{110\}$ sichtbar.

Ref.: G. Bartalini.

15. G. La Valle (in Messina): Krystallographische Ontersuchnng elniger organischer Verbindungen (Gazzetta chim. ital. 1896, 1, 200). Dargestellt von G. Errera.

1. Dibenzylcy anacetamid $\left(\mathrm{C}_{6} \mathrm{H}_{5} . \mathrm{CH}_{2}\right)_{2} \mathrm{C} . \mathrm{CN}$. $\mathrm{CONH}_{2}$.

Schmelzpunkt 1650. Krystalle aus Alkohol. Rhombisch.

$$
a: b: c=0,4280: 1: 0,3067 \text {. }
$$

Beobachtete Formen: $\left\{\begin{array}{lll}0 & 0\end{array}\right\},\left\{\begin{array}{lll}1 & 1\end{array}\right\}$. Farblose, durchsichtige, nach $\left\{\begin{array}{lll}0 & 0\end{array}\right\}$ tafelige Krystalle.

$$
\begin{aligned}
& \text { Winkel: Beobachtet: Berechnet: }
\end{aligned}
$$

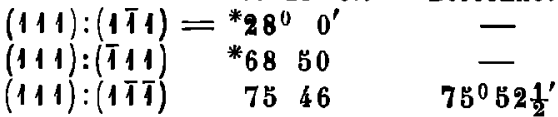

2. $\alpha$-Diäthylhydantoin $\left(\mathrm{C}_{2} \mathrm{H}_{5}\right)_{2} \mathrm{C}(\mathrm{CO} . \mathrm{NH})_{2}$.

Schmelzpunkt 1650. Krystalle aus Alkohol. Rhombisch.

$$
a: b: c=0,8391: 1: 2,5172 .
$$

Beob. Formen: $\{001\},\{011\},\{110\}$. Farblose, durchsichtige Krystalle.

$$
\begin{aligned}
& \text { Winkel: Beobachtet: Berechnet: }
\end{aligned}
$$

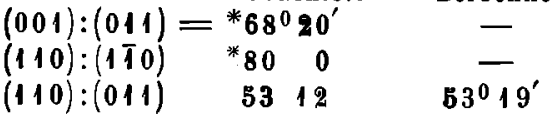

Vollkommene Spaltbarkeit nach $\{001\}$, auf welcher senkrecht die zweite Mittellinie zu stehen scheint.

3. Dimethylcyanacetamid $\left(\mathrm{CH}_{3}\right)_{2} \mathrm{C} . \mathrm{CN} . \mathrm{CONH}_{2}$.

Krystallsystem : Triklin.

$$
\begin{gathered}
a: b: c=1,0658: 1: 1,66251) . \\
\alpha=89^{\circ} 0^{\prime}, \beta=127^{\circ} 58^{\prime}, \gamma=107^{0} 54 \frac{1}{2}^{\prime} .
\end{gathered}
$$

1) Die Elemente sind im Original falsch berechnet.

Der Rof. 
Beobachlete Formen: $\{100\},\{010\},\{001\},\{110\},\{1 \overline{1} 0\},\{011\},\{1 \overline{1} 2\}$, $\left\{\begin{array}{l}0 \\ 0\end{array}\right\}$. Nach $\{001\}$ tafelige Krystalle.

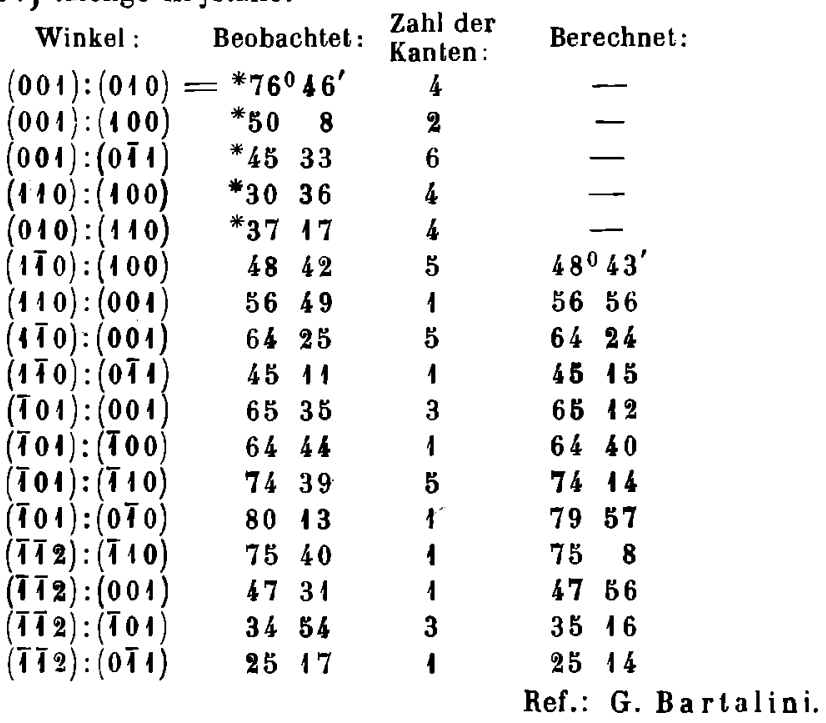

16. G. Boeris (in Mailand) : Krystallographische Untersuchung einiger organischer Substanzen (Gazzetla chim. ilal. 1896, 2, 297, 309, 342, 507, 520).

Nr. 1 und 2 dargestellt von G. Ciamician und P. Silber (siehe auch Ber. d. d. chem. Ges. 1896, 29, 1801).

1. Is o api ol (aus Dillöl) $C_{12} H_{14} O_{4}$.

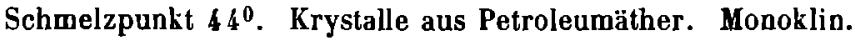

$$
a: b: c=0,8686: 1: 0,4610 ; \beta=79^{0} 30^{\prime} \text {. }
$$

Beobachtete Formen: $\{100\} ;\{110\},\{010\},\{111\},\{011\}$. Nach [001] prismatische Krystalle. Vorherrschend $\{100\},\{010\},\{111\},\{011\}$. Zwillinge nach $(100)$.

Winkel: Beobachtet: Zahl der Berechnet:

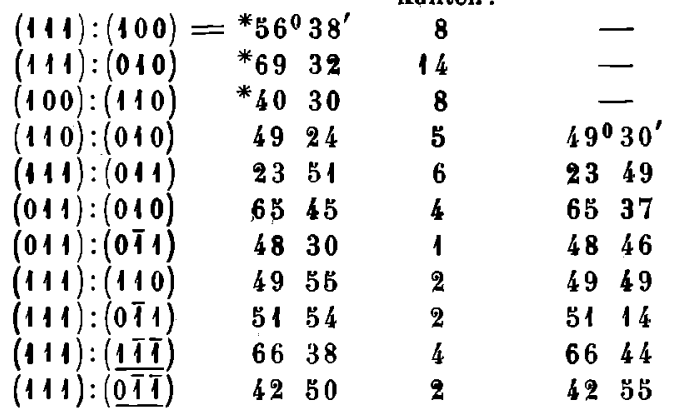

Optische Axenebene (010). Auf (010) bildet eine Schwingungsrichtung mil $\left[\begin{array}{ll}0 & 0\end{array}\right]$ im spitzen Winkel $\boldsymbol{\beta}$ einen Winkel von $32^{0} \mathrm{ca}$. Vollkommene Spaltbarkeil nach $\{010\}$. 
2. Tetramethylapionol $\mathrm{C}_{6} \mathrm{H}_{2}\left(\mathrm{OCH}_{3}\right)_{4}$.

Schmelzpunkt 890. Rhombisch.

$$
a: b: c=0,9454: 1: 1,0755 \text {. }
$$

Beobachlete Formen: $\{001\},\{101\},\{201\},\{110\},\{011\},\{021\},\{221\}$.

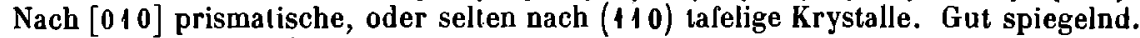
Zwillinge nach $\left\{\begin{array}{lll}1 & 1 & 0\end{array}\right\}$ erhält man mittelst der Gleitflächen.

Im Folgenden bezieht sich $\mathbf{A}$ auf ein aus » Apionol aus Dillöl « dargestelltes, B auf ein aus gewöhnlichem Apionol dargestelltes Präparat.

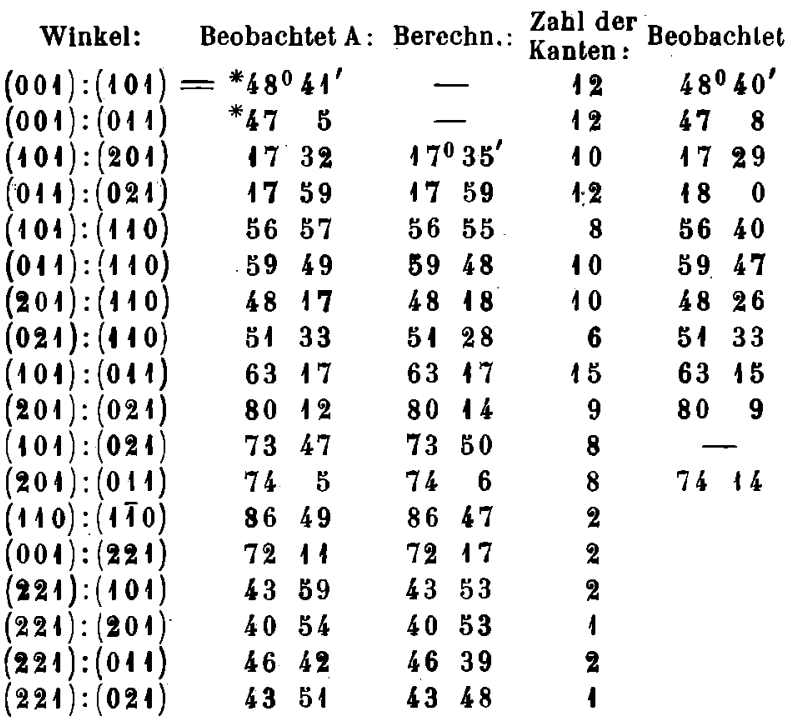

Optische Axenebene parallel zu $\{100\}$. Ersle Mittellinie posiliv und senkrecht zu $\{001\}$. An einem Prisma (021:001) wurde mittelst der Methode der Minimalablenkung $\beta=1,5439(\mathrm{Na})$ bestimml. An einem nach $\{001\}$ tafelartigen Krystalle wurde $2 E_{a}=80^{\circ} 1^{\prime}(\mathrm{Na})$ beobachtet. Hieraus erbielt man $2 V_{a}=$ $49^{\circ} 13^{\prime}(\mathrm{Na})$.

3. Phenylcumalinpikrat $\mathrm{C}_{11} \mathrm{H}_{8} \mathrm{O}_{2} \cdot \mathrm{C}_{6} \mathrm{H}_{2}\left(\mathrm{NO}_{2}\right)_{3} \mathrm{OH}$.

Dargestellt von F. Severini. Schmelzpunkt $81^{0}-82^{\circ}$. Krystalle aus Aether. Triklin.

$$
\begin{gathered}
a: b: c=1,3443: 1: 1,7030 . \\
\alpha=107^{\circ} 17^{\prime}, \beta=114^{\circ} 14^{\prime}, \gamma=63^{\circ} 30^{\prime} .
\end{gathered}
$$

\begin{tabular}{|c|c|c|c|}
\hline Winkel & Beobachtet: & $\begin{array}{l}\text { Zahl der } \\
\text { Kanten: }\end{array}$ & Berechnet \\
\hline$\left(\begin{array}{lll}1 & 0 & 0\end{array}\right):\left(\begin{array}{lll}0 & 0 & 1\end{array}\right)$ & $={ }^{*} 71^{0} \quad 2^{\prime}$ & 7 & - \\
\hline$(100):(010)$ & $* 11152$ & 3 & \\
\hline$\left(\begin{array}{lll}0 & 0 & 1\end{array}\right):\left(\begin{array}{lll}0 & 1 & 0\end{array}\right)$ & ${ }^{*} 8158$ & 4 & \\
\hline$(100):(110)$ & ${ }^{*} 6621$ & 2 & - \\
\hline$(001):(\overline{1} 01)$ & *66.19 & 7 & - \\
\hline$(001):(0 \overline{1} 0)$ & $97 \quad 69$ & 4 & $98^{0} 2^{\prime}$ \\
\hline
\end{tabular}

Beobachtete Formen: $\{100\},\{010\},\{001\},\{110\},\{\overline{1} 01\}$. Nach $\{001\}$ tafelige Krystalle. 


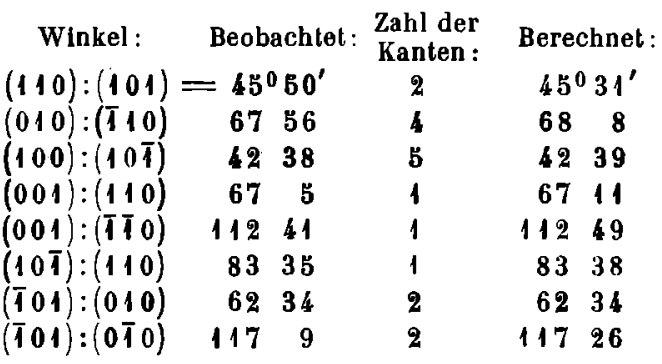

4. Is opernitros ofenkon $\mathrm{C}_{10} \mathrm{H}_{16} \mathrm{~N}_{2} \mathrm{O}_{2}$.

Dargestellt von E. Rimini. Schmelzpunkt $8^{\circ}$. Rhombisch.

$$
a: b: c=0,6775: 1: 0,4621 \text {. }
$$

Beobachtete Formen: $\{100\},\{110\},\{010\},\{011\}$. Nach $\{010\}$ tafelige Krystalle.

\begin{tabular}{|c|c|c|c|}
\hline Winkel: & Beobachtet: & $\begin{array}{l}\text { Zahl der } \\
\text { Kanten: }\end{array}$ & Berechnet \\
\hline$(00):(110)$ & ${ }^{*} 34^{0} \quad 7^{\prime}$ & 10 & 一 \\
\hline 10$):(011)$ & ${ }^{*} 650$ & 10 & - \\
\hline 11$):(110)$ & 7624 & 3 & $76^{0} 24^{\prime}$ \\
\hline
\end{tabular}

Spaltbarkeit nicht beobachtet. Ebene der oplischen Axen parallel zu $\{001\}$.

5. An ilderivat $\left(C_{14} \mathrm{H}_{19} \mathrm{NO}_{3}\right)$ der Säure $\mathrm{C}_{8} \mathrm{H}_{14} \mathrm{O}_{4}$, welche man durch Oxydation des Isokamphers erbält. Dargestellt von Rimini und Angeli.

Schmelzpunkt $160^{\circ}$. Krystalle aus wässerigem Alkohol. Rhombisch.

$$
a: b: c=0,9520: 1: 2,7383 \text {. }
$$

Beobachtete Formen: $\{001\},\{111\}$. Tafelig nach $\{001\}$.

$$
\begin{array}{cccc}
\text { Winkel : } & \text { Beobachtet: } & \begin{array}{c}
\text { Zahl der } \\
\text { Kanten : }
\end{array} & \text { Berechnel: } \\
(111):(111)={ }^{*} 89^{0} 14^{\prime} & 7 & - \\
(111):(11 \overline{1}) & * 2816 & 9 & - \\
(111):(111) & 8355 & 6 & 8^{0} 55^{\prime}
\end{array}
$$

Vollkommene Spaltbarkeit nach $\{001\}$. Oplische Axenebene (010). Erste Mitlellinie positiv und senkrecht zu $\{001\}$. Auf einer Spaltplatte wurde $2 E_{a}=$ $117^{0} 1 b^{\prime}(\mathrm{Na})$ beobachtet. Dispersion der optischen Axen sehr schwach, $\varrho>v$.

6. $\alpha$-Isopropylglularsäure-Anilderival von Perkin (chemische Zusammensetzung und Schmelzpunkt $=$ vor.).

Krystallsystem : Rhombisch.

$$
a: b: c=0,9440: 1: 2,6931 \text {. }
$$

Beobachtete Formen: $\{001\},\{111\}$. Tafelig nach $\left\{\begin{array}{lll}0 & 1\end{array}\right\}$.

$$
\begin{aligned}
& \text { Winkel: Beobachtet: } \begin{array}{c}
\text { Zahl der } \\
\text { Kanten: Berechnet: }
\end{array}
\end{aligned}
$$

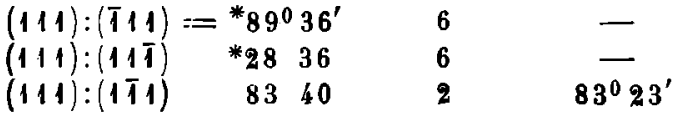

Vollkommene Spaltbarkeit nach $\{001\}$. Optische Axenebene parallel zu $\{010\}$. Erste Mittellinie positiv, senkrecht zu $\{001\}$. Auf einer Spaltplatte wurde 
$2 E_{a}=117^{0} 24^{\prime}$ gemessen. Dispersion der optischen Axen sehr schwach, $\varrho>v$. Hiernach sind die beiden letzlen Verbindungen auch krystallographisch identisch.

Ref.: G. Bartalini.

17. A. Ferro (in Genua): Ueber die Identitit des Formopyrins mit dem Methylendiantipyrin (Gazzetta chim. ital. 1896, 2, 411).

Das Formopyrin wurde bereits von Goguel (dargestellt von Marcourt) und das Methylendiantipyrin von Bartalini (dargestellt von G. Pellizzari) untersucht. Die Constanten des aus Antipyrinalloxan dargestellten Methylendiantipyrins sind :

$$
a: b: c=0,8344: 1: 0,7888 ; \beta=85^{0} 50 \frac{1}{2}^{\prime} \text {. }
$$

Die des aus Formaldehyd dargestellten Methylendiantipyrins (Pellizzari) sind

$$
a: b: c=0,8288: 1: 0,7886 ; \beta=85^{0} 48^{\prime} \text {. }
$$

Die des Formopyrins von Marcourt sind

$$
a: b: c=0,8252: 1: 0,7854 ; \beta=85^{0} 34 \frac{1}{2}^{\prime} .
$$

Und die Mittel

$$
a: b: c=0,8285: 1: 0,7876 ; \beta=85^{0} 44^{\prime} \text {, }
$$

welche sehr nahe den von Goguel und Bartalini gefundenen Werthen stehen (s. diese Zeitschr. 27, 543).

Ref.: G. Bartalini.

18. L. Brugnatelli (in Pavia): Ueber einige neue Sulfonverbindungen (Rivista di Min. e Crist. ital. di Panebianco 1896, 15, 53). Dargestellt von R. Otto.

$$
\text { 1. Phenylsulfonanilid } \mathrm{C}_{6} \mathrm{H}_{5} \mathrm{SO}_{2} \cdot \mathrm{HN} \cdot \mathrm{C}_{6} \mathrm{H}_{5} \text {. }
$$

Schmelzpunkt $110^{\circ}$. Krystalle aus Essigäther. Tetragonal.

\begin{tabular}{|c|c|c|c|}
\hline Winkel: & Beobacht & $\begin{array}{l}\text { Zahl der } \\
\text { Kanten : }\end{array}$ & Berechnet \\
\hline$(111):(112)$ & $14^{0} 8$ & 7 & $14^{0} \quad 4^{\prime}$ \\
\hline$(1112):(\overline{1} 12)$ & $* 119$ & 7 & - \\
\hline$(112):(1 \overline{1} 2)$ & $75 \quad 14$ & 4 & 75 \\
\hline$(111):(11 \pi)$ & $32 \quad 39$ & 4 & 3245 \\
\hline
\end{tabular}

$$
a: c=1: 2,4065 \text {. }
$$

Beobachtete Formen: $\{001\},\{111\},\{112\}$. (111\} und $\{112\}$ stets vorhanden, $\{111\}$ untergeordnet, $\{001\}$ oft fehlend. Farblose, sehr glänzende Krystalle. Meist pyramidal, selten tafelig nach $\{112\}$.

Keine Spaltbarkeit. An einem Prisma (112):(1T2) $=60^{\circ} 57^{\prime}$ wurde $\omega=$ 1,5999 $(\mathrm{Na}), \varepsilon=1,6486(\mathrm{Na})$ gefunden. Also Doppelbrechung posiliv, die auch direct beobachtet wurde.

2. Phenylsulf onmethylanilid $\mathrm{C}_{6} \mathrm{H}_{5} \mathrm{SO}_{2} \mathrm{~N} \cdot \mathrm{C}_{6} \mathrm{H}_{5} \cdot \mathrm{CH}_{3}$.

Schmelzpunkt $79^{\circ}-80^{\circ}$. Krystalle aus Essigäther. Monoklin.

$$
a: b: c=2,6965: 1: 3,8618 ; \beta=79^{\circ} 45^{\prime} \text {. }
$$

Beobachtete Formen: $\{001\},\{100\},\{101\},\{T 01\},\{110\},\{112\},\{112\}$. Farblose, sehr glänzende Krystalle. Theils verlängert nach [0 010$]$, theils tafelig nach $\left\{\begin{array}{lll}0 & 0 & 1\end{array}\right\}$. 


\begin{tabular}{|c|c|c|c|c|c|}
\hline Winkel : & \multicolumn{2}{|c|}{ Beobachtet: } & $\begin{array}{l}\text { Zahl der } \\
\text { Kanten: }\end{array}$ & \multicolumn{2}{|c|}{ Berechnet: } \\
\hline$(100):(110)$ & $={ }^{*} 69^{\circ}$ & $21^{\prime}$ & 10 & - & - \\
\hline$(001):\left(\begin{array}{l}1 \\
0\end{array} 1\right)$ & *62 & 8 & 7 & - & - \\
\hline$\left(\begin{array}{lll}\overline{1} & 0 & 0\end{array}\right):\left(\begin{array}{lll}1 & 0 & 1\end{array}\right)$ & *38 & 7 & 7 & & 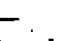 \\
\hline$\left(\begin{array}{lll}1 & 0 & 0\end{array}\right):\left(\begin{array}{lll}1 & 0 & 1\end{array}\right)$ & 31 & 17 & 7 & $3.1^{0}$ & $26^{\prime}$ \\
\hline$(001):(101)$ & 48 & 20 & 7 & 48 & 19 \\
\hline$(110):(101)$ & 72 & 26 & 6 & 72 & 29 \\
\hline$(110):(10 \pi)$ & 73 & $\mathbf{5 3}$ & 8 & 73 & $53 \frac{1}{2}$ \\
\hline$(001):(112)$ & 60 & 57 & 8 & 60 & 55 \\
\hline$\left(\begin{array}{lll}0 & 0 & 1\end{array}\right):\left(\begin{array}{lll}1 & 1 & 2\end{array}\right)$ & 66 & 44 & 6 & 66 & 42 \\
\hline$(100):(112)$ & 67 & 16 & 8 & 67 & 19 \\
\hline$(\overline{1} 00):(\overline{1} 12)$ & 75 & 54 & 8 & 75 & 53 \\
\hline$(1110):(1.12)$ & 25 & 30 & 6 & 25 & 30 \\
\hline$(110):(\bar{T} 12)$ & 43 & 54 & 7 & 43 & 58 \\
\hline$(110):(11 \overline{2})$ & 26 & 56 & 6 & 26 & 54 \\
\hline$(1110):(\overline{1} 1 \overline{2})$ & 50 & 55 & 8 & 50 . & 54 \\
\hline$(1001):(112)$ & 56 & 38 & 7 & 56 & 37 \\
\hline$(\bar{T} 01):(\bar{T} 12)$ & 62 & 8 & 7 & 62 & 9 \\
\hline$(112):(T 12)$ & 36 & 50 & 7 & 36 & 48 \\
\hline$(112):(\overline{1} 1 \overline{2})$ & 70 & 4 & 1 & 69 & 58 \\
\hline$(11 \overline{2}):(\overline{1} 12)$ & 61 & 9 & 1 & 61 & 6 \\
\hline
\end{tabular}

Vollkommene Spaltbarkeit nach $\{001\}$. Optische Axenebene parallel zu $\{010\}$. Durch die Spaltplatten tritt sehr geneigt eine optische Axe aus. Starke Doppelbrechung.

3. p-Tolylsulfonmelhylanilid $\mathrm{C}_{7} \mathrm{H}_{7} \mathrm{SO}_{2} \mathrm{~N}_{2} \mathrm{C}_{6} \mathrm{H}_{5} \cdot \mathrm{CH}_{3}$.

Schmelzpunkt 9 $4^{0}-95^{0}$. Krystalle aus Essigäther. Monoklin.

$$
a: b: c=2,4173: 1: 1,1363 ; \beta=87^{\circ} 6^{\prime} \text {. }
$$

Beobachtete Formen: $\{100\},\{101\},\{\overline{1} 01\},\{110\},\{011\},\{121\}$. Farblose, sehr glänzende Krystalle. Tafelig nach $\{100\}$ und verlängert nach [010]. \{1 21$\}$ schmal und selten.

Winkel: Beobachtet: Zahl der

\begin{tabular}{|c|c|c|}
\hline 100$):(110$ & ${ }^{*} 6$ & \\
\hline$(100):(101)$ & ${ }^{*} 62$ & 28 \\
\hline$(100):(10 \pi)$ & ${ }^{*} 67$ & 13 \\
\hline$(100):(011)$ & 88 & \\
\hline 101$):\left(\begin{array}{ll}1 & 0\end{array}\right)$ & 50 & 18 \\
\hline$(101):(011)$ & 53 & \\
\hline$(T 01):(011)$ & 53 & 27 \\
\hline$(101):(110)$ & 79 & 48 \\
\hline$(\bar{T} 01):(\bar{T} 10)$ & 81 & 27 \\
\hline$(110):(\bar{T} 10)$ & 44 & 58 \\
\hline$(110):(011)$ & 45 & 4 \\
\hline$(\overline{1} 10):(011)$ & 47 & 5 \\
\hline$\left(\begin{array}{lll}1 & 0 & 0\end{array}\right):\left(\begin{array}{lll}1 & 2 & 1\end{array}\right)$ & 78 & 16 \\
\hline$(101):(121)$ & 63 & 29 \\
\hline$(011):(121)$ & 19 & 39 \\
\hline$(110):(1$ & 25 & 2 \\
\hline 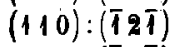 & 41 & 26 \\
\hline $21:(T 2 T)$ & b2 & $B 8$ \\
\hline
\end{tabular}
Kanten:

Berechnet: 
Vollkommene Spaltbarkeit nach $\{100\}$. Optische Axenebene parallel zu $\left\{\begin{array}{ll}0 & 0\end{array}\right\}$. Durch $\left\{\begin{array}{lll}1 & 0 & 0\end{array}\right\}$ und durch $\left\{\begin{array}{lll}1 & 0 & 1\end{array}\right\}$ tritt fast normal eine optische Axe aus. Die erste Mittellinie ist also im spitzen Winkel $\beta$.

$$
\text { 4. p-Tolylsulfonäthylanilid } \mathrm{C}_{7} \mathrm{H}_{7} \mathrm{SO}_{2} \mathrm{~N} \cdot \mathrm{C}_{6} \mathrm{H}_{5} \cdot \mathrm{C}_{2} \mathrm{H}_{5} \text {. }
$$

Schmelzpunkt $87^{0}-88^{\circ}$. Krystalle aus Essigäther. Rhombisch.

$$
a: b: c=0,377 b: 1: 1,5567 \text {. }
$$

Beobachtete Formen: $\{001\},\{011\},\{012\},\{111\}$. Weisse, wenig durchsichtige Krystalle; prismatisch nach [100]. Vorherrschend $\left\{\begin{array}{lll}0 & 1 & 1\end{array}\right\}$.

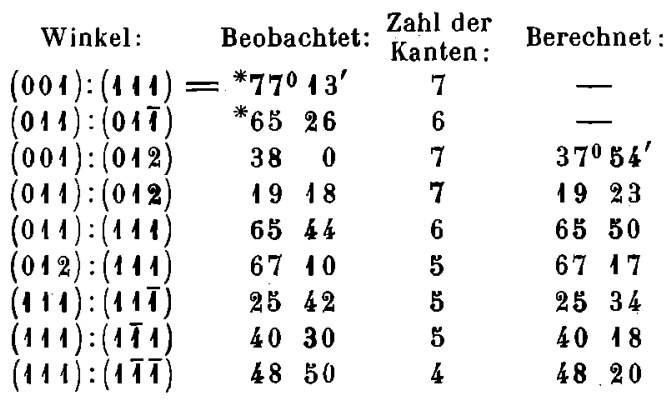

Vollkommene Spaltbarkeit nach $\{001\}$. Optische Axenebene parallel $\{010\}$. Erste Mittellinie parallel zu [100]. Starke und positive Doppelbrechung.

Ref.: G. Bartalini.

19. R. Panebianco (in Padua): Uober die Doppelbrechung der Seide (Rivista di Min. e Crist. ital. di Panebianco $1896,15,57$ ).

Nach den Arbeiten von D. M. Rossinski und von L. Vignon, die offenbar unrichtig sind, hat der Verf. von Neuem die Seide optisch untersucht und die folgenden Resultate erhalten. 1) Die Seidenfäden sind doppeltbrechend. Eine Auslöschungsrichtung ist parallel der Längsrichtung. 2) Die Farben zwischen gekreuzten Nicols (Längsrichtung unter $45^{0}$ mit denselben) sind sehr lebhaft. 3) Die Fibroïnfäden sind zweiaxig; optische Axenebene parallel (?) der Fadenrichtung; erste Mittellinie positiv, ebenfalls parallel der Fadenrichtung. Optischer Axenwinkel klein, Dispersion $\varrho<v$. Keine Ringe oder Lemniscaten; nur Andeutung der Farben an den.Hyperbelscheiteln. 4) Sericin ist doppeltbrechend, aber weitere Untersuchung ist unmöglich. Ein Dünnschliff von "Seripterium " zeigt ebenso das Fibroïn zweiaxig und die Axenebene parallel der Streifungen des Dünnschliffes. Diese Streifungen sind vielleicht durch das Scheermesser gebildet, und dann die Zweiaxigkeit in der colloïdalen Substanz durch Druck hervorgebracht. Das Sericin giebt lebhafte Farben in concentrischen Ringen ; es ist zweiaxig; Axenebene parallel derjenigen des Fibroïns, die erste Mittellinie ist aber zur Plattenebene geneigt. $\varrho<v$. Schliesslich stellt der Verf. die Frage auf, ob Fibroïn und Sericin krystallinische oder colloïdale Substanzen sind, lässt dieselbe aber unentschieden.

Ref.: G. Bartalini.

20. G. B. Negri (in Genua) : Ueber die Krystallformen des Aragonits rom Monté Ramazzo (Ligurien) (Ebenda 15, 65).

Untersucht wurden Krystalle, von welchen nur 7 einfach, 21 mit Streifungen auf $\left\{\begin{array}{lll}0 & 1\end{array}\right\}$ und $\{010\}$ entsprechend dem $Z$ willingsgesetz nach $\{110\}, 10$ ViellingsGroth, Zeitscluift f. Krystallogr. XXX. 
krystalle und 7 centrische $Z$ willinge sind. Stets prismatisch nach [001] und nur an einem Ende begrenzt. Die Fläche $(010)$ stets anwesend und breit und $(0 \overline{0} 0)$ oft fehlend, was mit der ungleichen Entwickelung von anderen Flächen den Anschein von Hemimorphie giebt.

Beobachtete Formen: $\{010\},\{041\},\{073\}^{*},\{031\},\{052\},\{021\},\{011\}$, $\{012\},\{430\}^{*},\{110\},\{570\}^{*},\{331\}^{*},\{111\},\{512\}^{*},\{9.2 .12\}^{*},\{413\}^{*}$, $\{3.2 .12\}^{*},\{431\}^{*},\{341\},\{342\}^{*},\{572\}^{*},\{7.10 .3\}^{*},\{231\}^{*},\{352\} *,\{121\}$, $\{132\},\{133\}^{*},\{271\}^{*},\{24.25 .1\}^{*}$.

Die mit Sternchen bezeichneten Formen sind für den Aragonit neu. Die Constanten wurden mittelst der Methode der kleinsten Quadrate berechnet.

$$
a: b: c=0,6227: 1: 0,7207 \text {. }
$$

Die Winkelwerthe der neuen Formen sind:

$$
\begin{aligned}
& \text { Winkel: Beobachtet: Zahl der Berechnet: }
\end{aligned}
$$

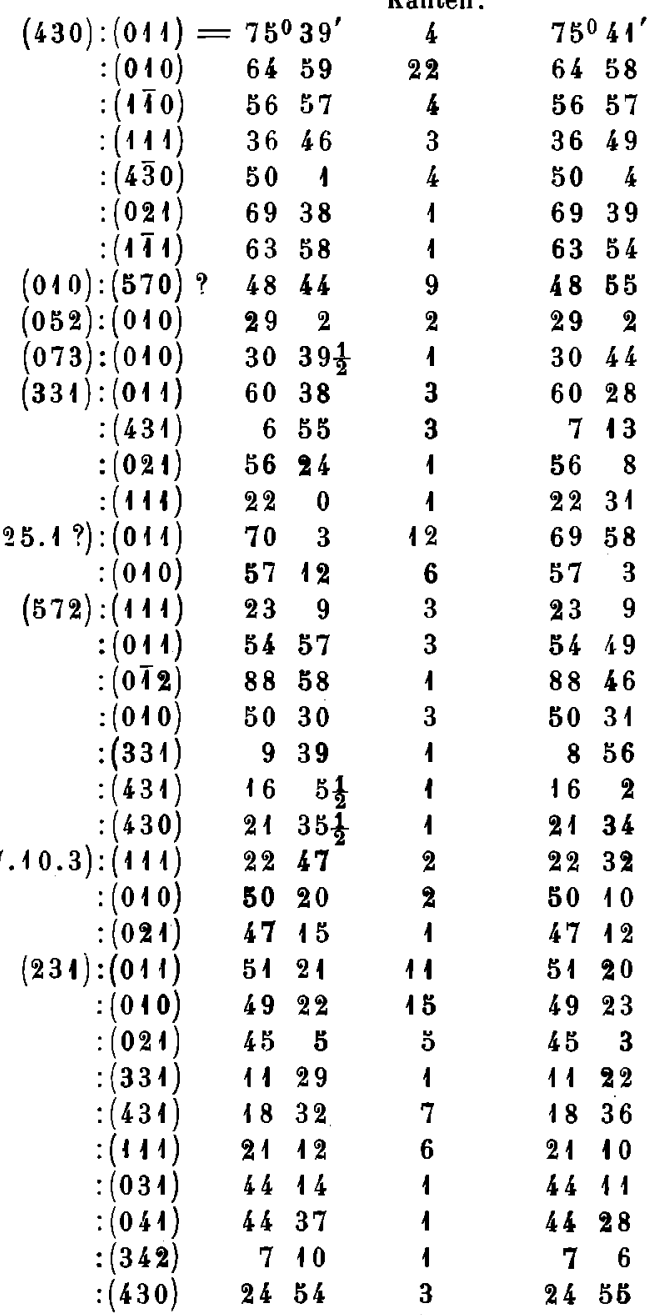


Winkel: Beobachtet: $\begin{aligned} & \text { Zahl der } \\ & \text { Kanten: Berechnet: }\end{aligned}$

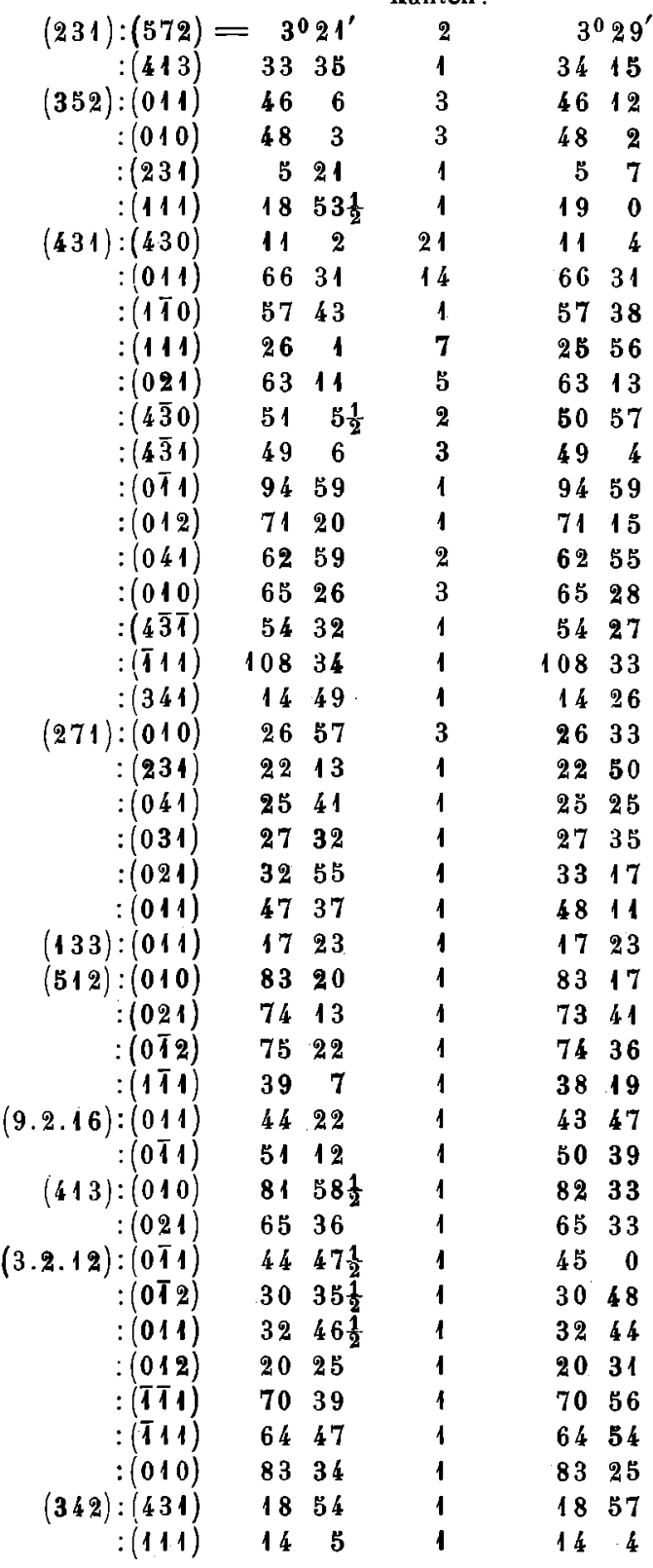

$\{430\},\{331\},\{572\},\{231\},\{352\},\{431\},\{271\},\{133\}$ wurden an vielen Krystallen beobachtet, die anderen neuen Formen aber je nur an einem Krystalle und im Allgemeinen mit nicht gut spiegelnden Flächen.

Ref.: G. Bartalini. 
21. A. Righi (in Bologna): Eeber die Auslöschungsrichtungen der elektrischen Wellen in Gypskrystallen (Rivista di Min. e Crist. ital. di P a n e b ian c o $1896,16,3)$.

In einer früheren Abhandlung (diese Zeitschr. 28, 185) hat der Verf. angegeben, dass die Auslöschungsrichtungen für die elektrischen Wellen in Gyps fast parallel (auf einer Platte $\|\left\{\begin{array}{lll}0 & 0 & 0\end{array}\right)$ zur (nicht faserigen) Spaltbarkeil $\left\{\begin{array}{lll}1 & 0 & 0\end{array}\right\}$ seien. Nun hat er den Winkel mit derselben Richtung gemessen und gefunden $1,3^{0}$ und $65,7^{0}$ mit der faserigen Spaltbarkeit, also $39,3^{\circ}$ mit der optischen Auslöschungsrichtung (mittlere Wellenlänge). Die elektrische Wellenlänge war 10,6 cm. Mit kleinerer Wellenlänge $(2,6 \mathrm{~cm})$ wurden genaue Resultate nicht erhalten. Für längere Wellen wären grössere Gypskrystalle nöthig.

Ref.: G. Bartalini.

22. E. Artini (in Mailand): Baryt von Vassera (Varese) (Ebenda 16, 10 , auch Atti d. Soc. Ital. d. sc. nat. 35).

Die begleitenden Mineralien sind: Galenit, Pyrit, Quarz, Fluorit. Auf dem letzteren wurden die folgenden Messungen gemacht: $(100):(920)=12^{0} 36^{\prime}$, berechn. $42^{0} 32^{\prime} ;(311):(131)=50^{0} 28^{\prime}$ berechn. $50^{\circ} 29^{\prime}$. An dem Baryt wurden die folgenden Formen beobachtet:

$\{100\},\{010\},\{001\},\{011\},\{101\},\{102\},\{210\},\{320\},\{110\},\{230\}$, $\{130\},\{111\},\{223\},\{112\},\{113\},\{114\},\{115\},\{122$.$\} Tafelig nach \{001\}$.

Vorherrschend $\left\{\begin{array}{lll}0 & 0 & 1\end{array}\right\}$ (schlecht spiegelnd), $\left\{\begin{array}{lll}0 & 0\end{array}\right\},\left\{\begin{array}{ll}0 & 1\end{array}\right\},\{111\},\{130\},\{111\}$.

Das Axenverhältniss $a: b: c=0,8126: 1: 1,3116$ wurde aus den folgenden Winkeln berechnet:

$$
\begin{aligned}
& (011):(010)=37^{0} 19^{\prime} \\
& (111):(011)=44 \quad 23
\end{aligned}
$$

Es folgt eine lange Reihe von Winkeln; da aber keine der Formen neu ist, so ist die Wiedergabe hier nicht erforderlich.

Ref.: G. Bartalini.

23. Derselbe: Ueber einige Mineralien von Gorno (Ebenda 16, 19, auch Alti d. Soc. Ital. sc. nat. 35).

Seltene oder nicht gut krystallisirte Mineralien dieses Fundortes sind: Galenit, Sphalerit $(\{111\}\{1 \overline{1} 1\}\{110\}$, Zwillingskrystalle nach [111], Smithisonit, Hydrozinkit, Pyrit $\{210\}$. Die messbaren Mineralien sind die folgenden:

1. Calamin. $0,1-5 \mathrm{~mm}$ gr. Krystalle, theils farblos und durchsichtig, theils gelblich und trübe, prismalisch nach [ $\left.\begin{array}{lll}0 & 0 & 1\end{array}\right]$. Fast immer mit dem antilogen Pole aufgewacbsen, oder Zwillinge nach $\left\{\begin{array}{lll}0 & 0 & 1\end{array}\right\}$ mit den beiden analogen Polen an den freien Enden. $\left\{\begin{array}{lll}0 & 1 & 0\end{array}\right\}$ sehr entwickelt und nach [ $\left.\begin{array}{lll}0 & 0 & 1\end{array}\right]$ gestreift.

Beobachtete Formen $\{010\},\{001\},\{110\},\{011\},\{031\},\{101\},\{503\}^{*}$, $\{301\}$. Vorherrschend und stets vorhanden $\{011\},\{101\},\{110\},\{010\}$. $(301):(503)=15^{0} 44^{\prime}$, berechnet $15^{0} 52^{\prime}$.

2. Cerussit. Dimensionen wie diejenigen von Calamin. Beobachtete Formen: $\{010\},\{012\},\{011\},\{032\},\{021\},\{031\},\{041\},\{051\},\{061\},\{0.13 .1\}$, $\{110\},\{130\},\{111\},\{113\},\{102\}$ ?. Letztere bestimmt durch die Zonen $[111: 113],\left[1 T_{1}: 113\right]$. Weisse und glänzende Krystalle. $\{010\}$ stets vorhanden und nach $[100]$ gestreift. $\{021\},\{012\},\{110\},\{111\},\{113\}$ sehr bäufig, $\{0.13 .1\}$ nur einmal beobachtet. Zwillingskrystalle nach $\{110\}$; auch Drillingskrystalle. 


\begin{tabular}{|c|c|c|c|c|c|}
\hline Winkel & Beob & achtet: & $\begin{array}{l}\text { Zahl der } \\
\text { Kanten: }\end{array}$ & \multicolumn{2}{|c|}{ Berecbnet } \\
\hline$(0.13 .1):\left(\begin{array}{lll}0 & 1 & 0\end{array}\right)$ & $=$ & $1^{\prime}$ & 1 & & $4^{\prime}$ \\
\hline$(010):(0 \overline{0} 0)$ & 62 & 46 & 2 & 62 & 47 \\
\hline$\left(\begin{array}{lll}1 & 10\end{array}\right):(\overline{T T 0})$ & 54 & 31 & 3 & 54 & 27 \\
\hline$(1100):(\overline{0 \bar{T} 0})$ & 4 & $7 \frac{1}{2}$ & 4 & 4 & 10 \\
\hline$(012):(\overline{0 \top \overline{2}})$ & 20 & $22^{2}$ & 4 & 20 & 24 \\
\hline$(111):(\overline{1 T})$ & 43 & $36 \frac{1}{2}$ & 2 & 43 & 34 \\
\hline$(113):(\overline{1} \overline{3})$ & 22 & 6 & 1 & 22 & 9 \\
\hline$(1 \overline{1} 1):(\overline{1} \overline{1} \overline{1})$ & 108 & 32 & 1 & 108 & 27 \\
\hline$(1 \overline{1} 3):(\overline{1} \overline{13})$ & 49 & 36 & 1 & 49 & 39 \\
\hline$(111):(0 \overline{2} \overline{1})$ & 3 & 30 & 3 & 3 & 35 \\
\hline
\end{tabular}

3. Wulfenit. Beobachtete Formen: $\{001\},\{101\},\{111\},\{113\} .\{5.1 .75\} *$ ? Kleine seltene, braune oder braungelbe durchsichtige Krystalle. Tafelig nach $\{001\}$. Vorherrschend $\{001\},\{111\},\{113\}$. $\{5.1 .75\}$ ? mit gekrümmten Flächen, aber recht häufig.

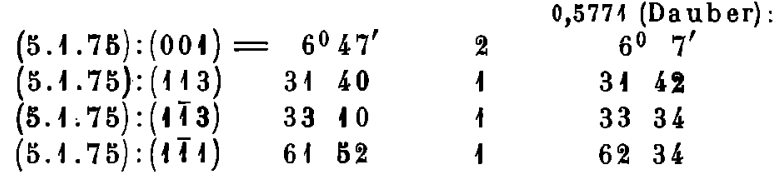

4. Calcit. Dimensionen 2-6 mm. Beobachlete Formen: $\{111\},\{100\}$, $\{3 \bar{T} T\},\{110\},\{11 \bar{T}\}$. Vorherrschend $\{11 \bar{T}\}$, aber mit unvollkommenen Flächen. Ebenso unvollkommen $\{111\}$. $\{100\}$ und $\{110\}$ nicht sehr breit, aber gut spiegelnd. Untergeordnel und oft fehlend $\{3 \bar{T} \bar{T}\}$.

Ref. : G. Bartalini.

24. R. Panebianco (in Padua): Ueber die Producte der Behandlang von Fibroïn und Sericin mit HCl (Ebenda 16, 29).

Reines Fibrö̈n in $\mathrm{HCl}$ gelöst, mil $\mathrm{NH}_{3}$ neutralisirt, gab nach Elimination des $\mathrm{NH}_{4} \mathrm{Cl}$ einen faserigen Niederschlag, welcher durch Pikrocarmin gelb gefärbt wurde. Die nadelförmigen Krystalle zeigten parallele Auslöschung, deren sphärische Aggregate ein regelmässiges schwarzes Kreuz zwischen gekreuzten Nicols. Hieraus scheint hervorzugehen, dass die Kryställchen $(0,0015-0,0005$ dick und $0,01 \mathrm{lang}$ ) zweiaxig mit der Längsrichtung parallel der ersten Mittellinie sind. In der That zeigen sie Hyperbeln mit der Reihenfolge (von aussen) der Farben Gelb, Roth, Violett (zweite Mittellinie). Das Sericin wurde wie Fibroïn untersucht, aber keine deutlichen Erscheinungen erhalten. Der Niederschlag durch Zufügung von Alkohol in der Lösung in $\mathrm{HCl}$ gab Kryställchen (0,003 mm lang) der tetragonalen Combinationen $\{111\},\{111\}\{100\},\{111\}\{100\}\{110\}$. Auf $\{111\}$ oder $\{100\}$ Auslöschungsrichtung parallel zu $[111: 11 \overline{1}]$ resp. [100:010]. Die seltenen Krystalle mit [001] vertical zeigen sich einfachbrechend. Mit Pikrocarmin nehmen sie eine almandinrothe Farbe an. Das und die anderen Eigenschaften können zur Erkennung von Fibroïn und Sericin dienen.

Ref.: G. Bartalini.

25. R. Penebianco (in Padua): Notiz uber krystallographische Morphologle (Ebenda 16, 33).

Der Verf. giebt die Formeln zur Berechnung der ebenen Winkel der Krystallkanten, welche für die mikroskopische Untersuchung und für die Construction 
der Krystallmodellnetze dienen. Die Grundlage des Beweises ist die folgende: 1) Die Projection eines beliebigen Polygons auf eine gerade Linie ist gleich Null.

2) Die orthogonale Projection einer geraden Linie ist gleich ihrer Länge, mit dem Cosinus des Winkels, welche sie mit der Projectionslinie bildet, mulliplicirt. Für das trikline System gilt die Formel :

worin

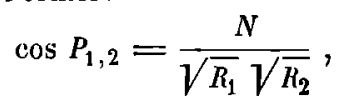

$$
\begin{aligned}
& N=a^{2} u_{1} u_{2}+b^{2} v_{1} v_{2}+c^{2} w_{1} w_{2}+\left\{\begin{array}{c}
G\left(u_{1} v_{2}+v_{1} u_{2}\right) \\
H\left(v_{1} w_{2}+w_{1} v_{2}\right) \\
I\left(w_{1} u_{2}+u_{1} w_{2}\right)
\end{array}\right. \\
& R_{1}=a^{2} u_{1}^{2}+b^{2} v_{1}^{2}+c^{2} w_{1}^{2}+2\left\{\begin{array}{l}
u_{1} v_{1} \cdot G \\
v_{1} w_{1} \cdot H \\
w_{1} u_{1} \cdot I
\end{array}\right. \\
& R_{2}=a^{2} u_{2}^{2}+b^{2} v_{2}^{2}+c^{2} w_{2}^{2}+2\left\{\begin{array}{l}
u_{2} v_{2} \cdot G \\
v_{2} w_{2} \cdot H \\
w_{2} u_{2} \cdot I
\end{array}\right. \\
& \begin{array}{l}
G=a b \cos \gamma \\
H=b c \cos \alpha \quad \text { oder } \\
I=c a \cos \beta
\end{array} \quad\left\{\begin{array}{l}
G=\frac{\cos C-\cos A \cos B}{\sin A \sin B} \cdot a b \\
H=\frac{\cos A-\cos B \cos C}{\sin B \sin C} \cdot b c \\
I=\frac{\cos B-\cos A \cos C}{\sin A \sin C} \cdot c a
\end{array}\right.
\end{aligned}
$$

wo der Kantenwinkel $P_{1}, 2$ der ebene Winkel der Kanten $\left[u_{1} v_{1} w_{1}\right]$, $\left[v_{2} u_{2} w_{2}\right]$, $a, b, c$ die Axeneinheiten, $\alpha, \beta, \gamma$ die Axenwinkel und $A, B, C$ die Winkel der Coordinatenebenen sind.

Aus derselben Formel ist leicht diejenige für das monokline Krystallsystem abzuleiten, indem man $\cos \alpha=\cos \gamma=0$ setzt, u. s. w. für die übrigen Systeme.

$$
\text { Ref.: G. Bartalini. }
$$

26. L. Vaccari (in Padua): Notizen zur venetianischen Mineralogie (Rivista di Min. e Crist. ital. di Panebianco 1896, 16, 93).

1. Ein gelber Zirkonkrystall von Novale, dessen Dimensionen $8 \times 6,5 \times$ $13,5 \mathrm{~mm}$ sind, zeigt die Combination $\{110\}\{111\}$ mit den Winkeln $(111):(111)=$ $56^{0} 45^{\prime},(111):(110)=47^{0} 56^{\prime}$. Spec. Gew. 4,65.

2. Baryt von S. Luca (Bassano). Er ist von Quarz begleitet und zeigt die folgenden Combinationen: $\left\{\begin{array}{lll}1 & 1 & 0\end{array}\right\},\left\{\begin{array}{lll}1 & 0 & 1\end{array}\right\},\left\{\begin{array}{lll}0 & 1 & 1\end{array}\right\},\left\{\begin{array}{lll}0 & 0 & 1\end{array}\right\}$ und $\left\{\begin{array}{lll}1 & 1 & 0\end{array}\right\},\left\{\begin{array}{lll}1 & 0 & 1\end{array}\right\},\left\{\begin{array}{lll}0 & 1\end{array}\right\}$, $\{001\},\{100\}$.

3. Analcim von Molvena (Bassano). Er zeigt die Form $\{110\}$ (Winkel $60^{\circ}$ und $\left.59^{0} 59^{\prime}\right)$. Spec. Gew. 2,35. Schwach doppeltbrechend. Es wurde Wasser, $\mathrm{SiO}_{2}, \mathrm{Al}_{2} \mathrm{O}_{3}$ und $\mathrm{Na}_{2} \mathrm{O}$ nachgewiesen.

Ref.: G. Bartalini.

27. D. Pantanelli (in Modena): Gedlegenes Kapfer nad Quecksilber im omillanischen Apennin (Alti della R. Accad. dei Lincei 1896, 5. Serie. Rendic. d. Cl. di Sc. Fis. etc., 1. sem., 11).

Das gediegene Kupfer in eocänen Schieferthonen und nicht im Zusammenhange mit Drusen oder Serpentingesteinen war bereits früher bekannt und 
besonders 1895 von Bentivoglio bei Cargedolo und Ospitaletto elc. Der Verf. hat Kupfer ebenso im Monte Galbone (Viano, Tresinarothal) gefunden. Oefters ist es in Malachit und Azurit (auch krystallisirt) umgewandelt. Es findel sich in Platten bis zum Gewichte von $10 \mathrm{~g}$, entweder gänzlich in Kupfercarbonat verwandelt oder mit einem inneren Kerne einer Mischung von $\mathrm{Cu}, \mathrm{CuO}, \mathrm{Fe}_{2} \mathrm{O}_{3}$ und Kieselsäure. Sehr selten findet man Stücke von gediegenem Kupfer; nie Spuren von Schwefelkupfer. Die Kupfererze findet man nur an einzelnen Orten. Nach dem Verf. ist die Entstehung dieses Minerals auf Sulfate, nach der Theorie von Knop, zurückzuführen.

Gediegenes Quecksilber. Es wurde im September 1895 in den Fundamenten eines Hauses im Lehmboden gefunden. Der Verf. stellte fest, dass der quecksilberballige Lehm in einer Schicht von ca. $15 \mathrm{~cm}$ zwischen zwei härteren, $45^{0}$ nach NO einfallenden Lehmschichten eingeschlossen ist. $\mathrm{Er}$ entbielt kleine Tropfen $\mathrm{Hg}$ mit Spuren $\mathrm{Ag}$ in $\mathrm{HgCl}$ eingeschlossen, welches man auch ohne gediegenes Quecksilber findet. Das letztere scheint aus der Reduction von $\mathrm{HgCl}$ entstanden zu sein.

Ref.: G. Bartalini.

28. D. Lovisato (in Cagliari) : Der Granat von Caprera und Sardinien (Ebenda 56).

Der Verf. giebt ein langes Verzeichniss von Fundorten des Granats auf Sardinien, welche aber last alle bereils bekannt waren. Er beschreibt besonders den Granat von Caprera, welcher von Turmalin (siehe diese Zeitschr. 28, 183) begleitet ist. Dimensionen $0,25-1 \mathrm{~mm}$. Amethyst- oder weinrothe Farbe. Er zeigt die Form $\{211\}$ und selten $\{110\}$, nie die Combination derselben. Die Krystalle sind in Quarz und selter in Feldspath oder Glimmer eingeschlossen. Das spec. Gew. ist 4,1016 (Mittel von 5 Beobachtungen). Die Analyse von M. Fasolo ergab:

I.

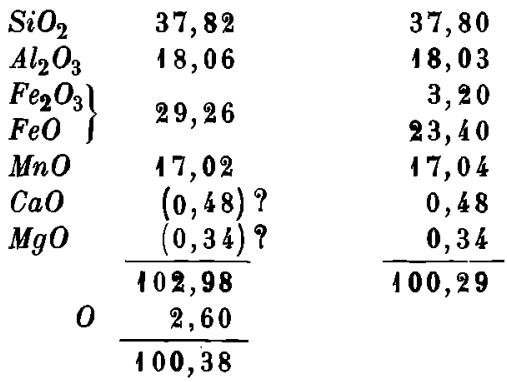

Aus II. folgt die Formel $\left.\begin{array}{c}(\mathrm{Fe}, \mathrm{Mn})_{3} \\ \mathrm{Al}_{2}\end{array}\right\} \mathrm{Si}_{3} \mathrm{O}_{12}$, in welcher ein kleiner Theil des $\mathrm{Mn}$ durch $\mathrm{Ca}$ und $\mathrm{Mg}$ und etwas $\mathrm{Al}_{2} \mathrm{O}_{3}$ durch $\mathrm{Fe}_{2} \mathrm{O}_{3}$ ersetzt ist. Der Ueberschuss der Kieselsäure zeigt eine kleine Menge von Quarz im Material der Analyse an. Alkalien wurden nicht, selbst nicht durch das Spectroskop, gefunden. Also wäre der Granat von Caprera ein Spessartin.

Ref.: G. Bartalini.

29. E. Artini (in Mailand): Krystellographische and optische Untersuchnng der Dimethylasparaginsänre (oder Dimethylamidobernsteinsäure) (Ebenda 1. sem., 458). - Dargestellt von Körner und Menozzi. Schmelzpunkt $185^{\circ}$. Krystalle aus Wasser. Monoklin. 


$$
a: b: c=1,2141: 1: 0,6340 ; \beta=78^{\circ} 19^{\prime} .
$$

Beobachtete Formen: $\{100\},\{001\},\{110\},\{\overline{2} 01\},\{011\},\{\overline{2} 11\}$. Farblose, wasserhelle, nach [ 001 1] prismatische Krystalle.

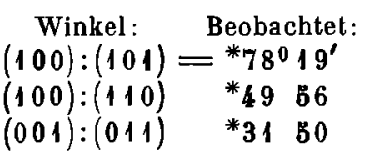

Vollkommene Spaltbarkeit nach $\{\overline{2} 01\}$. Optische Axenebene $\{010\}$.

Ref.: G. Bartalini.

30. L. Fantappiè (in Viterbo): Ein merkwürdiges Mineralvorkommen aus der Provinz Rom (Atti della R. Accad. dei Lincei 1896, 5. Serie. Rendic. d. Cl. di Sc. Fis. etc., 2. sem., 108).

In einem, hauptsächlich aus Feldspath bestehenden, ausserdem Hornblende und Glimmer enthaltenden erratischen Blocke der Ciminischen Gegend wurden in Höhlungen gut ausgebildete Krystalle gefunden, von welchen die folgenden merkwürdig sind:

1. D a n bu rit. Dimensionen $\frac{1}{2}-1 \frac{1}{2} \times 2-4 \mathrm{~mm}$. Im Allgemeinen Parallelverwachsungen, selten einzelne Krystalle, welche die folgenden Formen zeigen: $\{110\},\{120\},\{101\},\{041\},\{001\},\{010\}$. Vorherrschend $\left.\{120\},\left\{\begin{array}{lll}0 & 01\end{array}\right\} ; 010\right\}$ sehr schmal. Der Verf. giebt nur die folgenden Winkel :

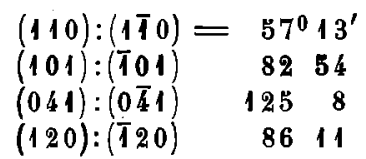

Letzterer liegt zwischen $85^{0} 8^{\prime}$ (Dana) des Danburits und $86^{\circ} 49^{\prime}$ des Topas. Durchsichtige oder durchscheinende, glasglänzende, farblose oder weingelbe Krystalle. Die Flächen sind oft schillernd, besonders $\{110\}$; die Ursache davon ist die Zersetzung durch die vulcanische Thätigkeit, da fast alle Mineralien dieses Vorkommens irisirend sind. Reaction auf $B a$.

2. Davyn. Dimensionen $\frac{2}{3} \times 1 \frac{1}{2} \mathrm{~mm}$. Beobachtete Formen: $\{111\},\{711$, $55 T\},\{5 T T, 11 T\},\{2 T T\}$. Die Flächen des Prismas sind senkrecht zu [111] gestreift. Glasglanz. Weisse, undurchsichtige oder halbdurchscheinende Krystalle.

In demselben Blocke findet man auch honiggelben Granat und Magnetit. In einem anderen Blocke wurden gefunden:

3. Quarz. Dimensionen $\frac{1}{2}-3 \frac{1}{2} \times \frac{2}{3}-4 \frac{1}{2} \mathrm{~mm}$. Er zeigt die gewöhnlichen Formen $\{100\},\{22 T\},\{2 T T\}$.

4. Turmalin. Dimensionen $\frac{1}{3} \times 2 \mathrm{~mm}$. Beobachtete Formen: $\{10 \mathrm{~T}\}$, $\{100\},\{11 T\}$. Die unregelmässige Entwickelung der Flïchen macht den Habilus der Krystalle ähnlich dem der Hornblende. Glas- bis Harzglanz. Pleochroïsmus sehr stark, braungelb und röthlich. Isomorphe Schichlung mit verschiedenen Farben; die kleineren Krystalle zeigen ein grünes Ende.

Ref.: G. Bartalini.

31. G. D'Achiardi (in Pisa): Der Granat von Affacata (Insel Elba) (Annali delle Università Toscane 20).

Beobachtete Formen: $\{111\},\{110\},\{211\},\{210\},\{100\}$ ?. Die beiden letzteren sind für diesen Fundort neu, aber $\left\{\begin{array}{lll}1 & 0 & 0\end{array}\right\}$ unsicher. Härte ein klein wenig 
höher als 7. Spec. Gew. 3,354--3,368. Schwache fleischrothe oder grünliche Farbe. Mit mehreren, nach yerschiedenen Richtungen geschnittenen Prismen wurden die folgenden Brechungsexponenten beobachtet:

$\begin{array}{rccccc} & N a & \text { Roth: } & \text { Gelb: } & \text { Grün: } & \text { Indigo: } \\ \text { Prisma I : } & 1,7601 & 1,7485 & 1,7599 & 1,7655 & 1,7730 \\ \text { - II : } & 1,7557 & 1,7550 & 1,7560 & 1,7626 & 1,7751\end{array}$

Es war unmöglich zu bestimmen, ob die Prismen zwei Bilder geben, wegen der geringen Durchsichtigkeit. Mit dem Babinet'schen Compensator wurde aber erhalten $\gamma-\beta=0,00092-0,00112$. Ferner führte der Verf. die optische Untersuchung einiger Platten aus, welche parallel den Flächen von $\{110\},\{111\},\{100\}$ geschnitten waren. Es ergab sich, dass die Krystalle rhombendodekaëdrische Structur haben und aus rhombischen Individuen zusammengesetzt sind, deren zweite negative Mittellinie zu $\left\{\begin{array}{lll}1 & 1 & 0\end{array}\right\}$ senkrecht steht. Die zwölf Individuen sind aber von Subindividuen in nicht gleicher Orientirung gebildet.

$$
\text { Ref.: G. Bartalini. }
$$

32. Derselbe: Ueber den Elbaner Tarmalin, II. Theil (Atti della Soc. Tosc. di Sc. Naturali, Memorie 15).

Ueber den wichtigsten Theil dieser Arbeit d. h. die Brechungsexponenten wurde schon früher (diese Zeitschr. 26, 213) referirt. Nun fügt der Verf. hinzu: Härte, specifisches Gewicht, Farben und Pleochroïsmus der verschiedenen Varî́etäten, endlich die Veränderung der Farben durch die Temperatur. Aus den Interferenzbildern schliesst er, dass die oplischen Anomalien ausser von dem polysynthetischen Aufbau auch von Spannung herkommen. Die Schmelzfiguren (mit Wachs) auf Platten \| der Axe geben das Verhältniss der Ellipsen zwischen $\frac{1,1}{1}$ und $\frac{1,6}{1}$. Die Farbe der Krystalle zeigt von unten nach oben stets die Reihenfolge: Gelb, Braungelb, unteres Schwarz, Grüngelb, Gelbgrünlich, Rosa, farblos, Grünblau, Blau, oberes Schwarz, und durch pyroëlektrische Untersuchung wurde festgestellt, dass das obere Schwarz stets dem analogen Pole entspricht.

Ref.: G. Bartalini.

33. S. Bertolio (in Iglesias): Die Mineralien der Insel S. Pietro (Sardinien) (Bolletino del R. Com. Geol. d'Italia 1896, 405). - Die wichtigsten Mineralien dieses Fundortes sind: Feldspath (Anorthoklas, Oligoklas, Labradorit), Quarz, Opal, Tridymit, Amphibol (Arfvedsonit), Baryl, Pyrolusit.

Der Anorthoklas zeigt die Formen $\{110\}\{010\} ;$ sp. G. 2,58-2,59. Die Analyse ergab: $\mathrm{SiO}_{2}$ 66,1, $\mathrm{Al}_{2} \mathrm{O}_{3}$ 18,2, $\mathrm{CaO}$ 0,1, $\mathrm{K}_{2} \mathrm{O} 3,5, \mathrm{Na}_{2} \mathrm{O}$ 11,4.

Der Amphibol zeigt die Formen $\{110\},\{010\},\{111\},\{001\}$. Die Analyse ergab:

\begin{tabular}{lr}
$\mathrm{SiO}_{2}$ & 49,10 \\
$\mathrm{Al}_{2} \mathrm{O}_{3}$ & $\mathbf{5}, 50$ \\
$\mathrm{Fe}_{2} \mathrm{O}_{3}$ & 4,20 \\
$\mathrm{FeO}$ & 27,70 \\
$\mathrm{MnO}$ & $0, \mathbf{5 0}$ \\
$\mathrm{CaO}$ & 0,13 \\
$\mathrm{MgO}$ & 0,17 \\
$\mathrm{~K}_{2} \mathrm{O}$ & 1,60 \\
$\mathrm{Na}_{2} \mathrm{O}$ & $\mathbf{1 0 , 5 0}$ \\
\hline & 99,40
\end{tabular}


Die Analyse des nicht krystallisirlen Pyrolusits ergab:

\begin{tabular}{lr}
$\mathrm{MnO}_{2}$ & 36,0 \\
$\mathrm{MnO}$ & 4,0 \\
$\mathrm{Fe}_{2} \mathrm{O}_{3}$ & $1 \mathbf{5 , 0}$ \\
$\mathrm{Al}_{2} \mathrm{O}_{3}$ & $\mathbf{5 , 0}$ \\
$\mathrm{CaO}$ & 1,0 \\
$\mathrm{MgO}$ & 2,0 \\
Phosphorsäure & 0,4 \\
Kieselsäure & 9,1 \\
Combinationswasser & 6,3 \\
Schwefelsäure & 0,1 \\
Verlust zu $100^{\circ}$ & 20,5 \\
\cline { 2 - 2 } & $\mathbf{9 9 , 4}$
\end{tabular}

Dieses Mineral scheint aus im Wasser der Quellen gelöstem Mangancarbonat entstanden zu sein.

Ref.: G. Bartalini.

34. L. Colomba (in Turin): Mineralogische Beobachtungen tiber den Sand der Turiner Hügel (Atti d. R. Accad. d. Sc. di Torino 1895-1896, 31, 593). Derselbe: Ceber den Epidot ron Oulx and die ihn begleitenden Mineralien (Ebenda, 1890-1891, 26, 537).

Der zu Marentino bei Turin vorkommende Sand enthält mannigfache Mineralien, und einige derselben sind sehr wichtig, weil sie die Herkunft erkennen lassen. Die Körner sind wenig abgerundet. Die beobachteten Mineralien sind: 1) Quarz in durchsichtigen oder weisslichen Bruchstücken; 2) Feldspath in gerundeten und schmelzbaren Körnern, selten in zerbrochenen Albitzwillingen. 3) Talk, Chlorit, Serpentin und Pyroxen ohne wichtige Charaktere. 4) Unter den Glimmern ist der farblose oder grünliche Muscovit häufiger, Biotit seltener: 5) Epidot in mehr oder minder gerundeten, grünlichgelben Körnern, mit schwachem Pleochroïsmus. 6) Granat in Rhombendodekaëdern oder gerundeten Körnern. Die Farbe schwach roth oder gelblich. 7) Glaukophan fast immer in Bruchstücken mit nicht gerundeten Kanten, stark oder schwach blau (vielleicht von zweierlei Herkunft), Pleochroïsmus blau und violett (selten grüngelb und blau oder grüngelb und violett); an den dunkleren und grösseren Krystallen wurde $\left\{\begin{array}{lll}1 & 1 & 0\end{array}\right\}$ und selten $\left\{\begin{array}{lll}0 & 1 & 0\end{array}\right\}$ beobachtet; einige kleine Prismen mit der Form des Glaukophan sind grün, gebrochen und bestehen aus Chlorit; sie sind vielleicht Zersetzungsproducte des Glaukophans, wie die ähnlichen von Beaume (diese Zeitschr. 26, 216). 8) A mphibol sehr reichlich, besonders farbloser Tremolit $\{110\},\{010\}$ mit scharfen Kanten; auf $\left\{\begin{array}{lll}0 & 0 & 0\end{array}\right.$ Auslöschungsrichtung mit der Verlängerungsaxe $15^{0}$ bildend. Seltener ist eisenhaltiger, grünlicher Tremolit mit Auslöschungswinkel bis $18^{\circ}$ und schwachem Pleochroïsmus, ebenso der grasgrüne Aktinolith mit Auslöschungswinkel bis $22^{\circ}$. 9) Turmalin, wurde durch $\mathrm{HFl}$ getrenut, öfters in Krystallen mit deutlichem Hemimorphismus, bietet sich in vier Varietäten dar, welche durch den Pleochroïsmus unterschieden sind: braungelb und braun, farblos und braungelb, farblos und braungrün, hell braungrünlich und braunviolett. 10) $\mathrm{Magnetit}$ mit $\mathrm{SiO}_{2}$. 11) Pyrit zersetzt in Limonit. 12) Chromit in Körnern, welche Chromreaction geben. 13) Spinell, sehr gewöhnlich in nicht gerundeten rosenrothen Körnern oder in fast farblosen Oktaëdern. 14) Zirk on sehr gewöhnlich in kleinen, verlängerten und fast farblosen, scharfen Krystallen. 15) Rutil in gelben oder rothgelben verlängerten Nadeln, welche 
der Varietät Sagenit anzugehören scheinen. Einige Krystalle der Form $\{111\}$ mit charakteristischer Streifung scheinen Oktaëdrit zu sein. 16) Baryt selten in kleinen, gestreiften, farblosen Bruchstücken. 17) Menaccanit ziemlich selten in kleinen, nicht gerundeten, schwach oder nicht magnetischen Körnern.

Die Mineralien Glaukophan, Rutil, Oktaëdrit, Menaccanit, Baryt, Turmalin und theilweise Feldspath zeigen ähnliche Charaktere wie diejenigen von Beaume und im Allgemeinen wie diejenigen des oberen Thales der Dora Riparia und stammen wahrscheinlich aus diesem Thale her, über dessen Mineralvorkommen nur spärliche Nachrichen existiren, abgesehen von der oben citirten Beschreibung der Mineralien von Oulx. Da von dieser Arbeit des Verf. seiner Zeit in dieser Zeitschr. versehentlich kein Auszug gegeben wurde, so mögen die Angaben des Verf. hier nachgetragen werden.

Epidot. Dimensionen der Krystalle $1-10 \mathrm{~mm}$. Theilweise radialfaserig. Die grösseren Krystalle sind tief olivengrün, fast undurchsichtig mit stark gestreiften Flächen; die kleineren hell olivengelb, durchsichtig und mit genügend spiegelnden und messbaren Flächen. Sie zeigen die Formen: $\left\{\begin{array}{lll}10 & 0\end{array},\left\{\begin{array}{lll}0 & 0\end{array}\right\},\left\{\begin{array}{ll}0 & 1\end{array}\right\}\right.$, $\left\{\begin{array}{lll}1 & 01\end{array}\right\},\left\{\begin{array}{l}1 \\ 0\end{array} 1\right\},\{012\},\{201\},\{111\},\{011\},\{110\},\{103\}$ (Orientirung von Miller), von denen $\{001\},\{010\},\{100\},\{101\},\{102\}$ häufiger sind. Verlängert nach [010]. Die Analyse ergab:

I.

\begin{tabular}{cr}
$\mathrm{SiO}_{2}$ & 37,41 \\
$\mathrm{Al}_{2} \mathrm{O}_{3}$ & 20,56 \\
$\mathrm{Fe}_{2} \mathrm{O}_{3}$ & $\mathbf{1 5 , 2 2}$ \\
$\mathrm{CaO}$ & $\mathbf{2 4 , 0 1}$ \\
$\mathrm{MgO}$ & $\mathbf{0 , 4 5}$ \\
$\mathrm{MnO}$ & $\mathrm{Spur}$ \\
$\mathrm{CO}_{2}$ & Spur \\
Glühverlust & $\mathbf{1 , 9 7}$ \\
\cline { 2 - 2 } & $\mathbf{9 9 , 6 2}$
\end{tabular}

II.

37,56

20,23

15,39

23,98

0,52

Spur

Spur

$\frac{1,81}{99,49}$

Hieraus die Formel:

$$
\mathrm{H}_{2} \mathrm{O} . \mathrm{Fe}_{2} \mathrm{O}_{3} .2 \mathrm{Al}_{2} \mathrm{O}_{3} .4,5 \mathrm{CaO} .6,5 \mathrm{SiO}_{2} \text {. }
$$

Dieser Epidol ist jedoch nicht rein.

A mphibol (Aktinolith). Die Analyse des reineren Minerals ergab:

\begin{tabular}{lr}
$\mathrm{SiO}_{2}$ & $\mathbf{5 4 , 6 \mathrm { B }}$ \\
$\mathrm{Al}_{2} \mathrm{O}_{3}$ & 0,47 \\
$\mathrm{FeO}$ & 10,37 \\
$\mathrm{CaO}$ & 16,13 \\
$\mathrm{MgO}$ & 18,68 \\
\hline & 100,30
\end{tabular}

Hieraus ergiebt sich die Formel

$$
\mathrm{FeSiO}_{3} \cdot 3 \mathrm{MgSiO}_{3} \cdot 2 \mathrm{CaSiO}_{3} \text {. }
$$

Der Amphibol ist aber mit einem anderen braungelben Mineral gemischl und die Analyse desselben, wenn fast rein, ergiebt: 


\begin{tabular}{lr}
$\mathrm{SiO}_{2}$ & 46,17 \\
$\mathrm{Fe}_{2} \mathrm{O}_{3}$ & $\begin{array}{r}\mathrm{FeO} \\
\mathrm{Al} \mathrm{O}_{3}\end{array}$ \\
$\mathrm{MgO}$ & 8,51 \\
$\mathrm{CaO}$ & 12,92 \\
& 22,63 \\
\hline & 100,26
\end{tabular}

Dieses braungelbe Mineral scheint ein Zersetzungsproduct des Epidots und des Amphibols zu sein.

A lb it. Immer Zwillingskrystalle nach dem Albilgesetz.

Beobachtete Formen: $\{010\},\{130\},\{110\},\{0 \overline{2} 1\},\{001\},\{101\},\{\overline{1} 30\}$, $\left\{\begin{array}{lll}1 & 1 & 0\end{array}\right\}$ ?. Häufigere Formen $\{010\},\{001\},\{110\},\{101\}$. Oeflers grünlich durch Epidoteinschlüsse.

Die Analyse ergab:

\begin{tabular}{lrr} 
& \multicolumn{1}{c}{$\mathrm{I}}$. & \multicolumn{1}{c}{ II. } \\
$\mathrm{SiO}_{2}$ & 67,73 & $67, \mathbf{5 9}$ \\
$\mathrm{Fe}_{2} \mathrm{O}_{3}$ & 0,70 & 0,81 \\
$\mathrm{Al}_{2} \mathrm{O}_{3}$ & 16,91 & 17,16 \\
$\mathrm{MgO}$ & 0,18 & 0,10 \\
$\mathrm{CaO}$ & 3,81 & 3,78 \\
$\mathrm{~K} \mathrm{O}$ & 0,81 & 0,87 \\
$\mathrm{Na}_{2} \mathrm{O}$ & 9,21 & 9,11 \\
\cline { 2 - 3 } & $\mathbf{9 9 , 3 5}$ & 99,42
\end{tabular}

Ferner findet man häufig Calc it $(\{20 \bar{T}\}$ oder ein unbestimmbares, stumpfes Rhomboëder $), Q$ uarz $(\{100\},\{22 T\},\{2 \bar{T} T\}$ und selten $\{4 \overline{1} \overline{2}\})$, seltener Hämatil und Pyrit.

Ref.: G. Bartalini.

35. S. Franchi (in Rom): Ueber das Vorkommen des Lawsonits als Bestandtheil einiger italienischer Gesteine (Alti d. R. Accad. d. Sc. di Torino $1896-97,32,182)$.

In den Jahren 1893 und 94 fand der Verf. in einigen metamorphosirten diabasischen Gesteinen der Provinz von Cuneo ein Mineral in rhombischen Lamellen, welches zu bestimmen unmöglich war. 1895 fand er dasselbe Mineral in ähnlichen Gesteinen von anderen Fundorten, endlich auch in Gesteinen von Pegli (Riviera Ligure), von der Insel Giglio und in einem Gastalditgestein von Val Chianale. Aus dem letzteren wurden einige kleine Krystalle von $\frac{1}{4} \mathrm{~mm}$ isolirt ${ }^{1}$ ). Im Jahre 1896 fand Verf. im Vallone della Niera (Val Chianale) dasselbe Mineral in Krystallen von $1-2 \mathrm{~mm}$ in einigen Clorit-Aktinolithzonen mit blauem Amphibol. Prof. Spezia hielt das Mineral für Lawsonit. In der That stimmen die physikalischen Eigenschaften und die Lagerung fast überein, ebenso bestätigte eine vorläufige Analyse von Mattirolo die Sache. Ferner findet man dieses Mineral in Diabasporphyriten vom Val Maira von der Insel Giglio; in Gesteinen, welche aus blauem Amphibol (von metamorphosirten Diabasen) zusammengesetzt sind, und von Val Chisone, Maira, Grana, Valloriate, Pegli und der Insel Giglio. Endlich in Chlorit-Aktinolithzonen der Gesteine, welche der Verf. lawsonitführende Natron-Amphibolite nennt und die ebenso von Diabasen herstammen (Val Chianale, Maira, Grana, Valloriate). Danach wäre das Mineral als Pseudomorphose aus Plagioklasen entstanden.

Hef.: G. Bartalini.

1) S. Franchi, Prasiniti ed anfiboliti sodiche provenienti dalla metamorfosi di roccie diabasiche ecc. (Boll. della Soc. Geol. italiana 1896, 2). 
36. H. Goguel (in Bordeaux): Beitrng zur Kenntniss der krystallisirten Arseniate und Antimoniate (Contribulion à l'étude des arséniates et antimoniates crist. préparés per voie humide. Bordeaux 1894, 75 SS.).

Zur Darstellung der untersuchten arsensauren Salze wurde das betreffende Metall oder sein Oxyd in Arsensäure gelöst oder die Lösung des Acetats durch eine solche von Arsensäure zersetzl, der entstandene flockige Niederschlag durch einen kleinen Säureüberschuss gelöst und erwärmt, wobei sich erst eine Gallerte bildet, welche bei weiterem Erhitzen in den krystallisirten Zustand übergeht. Die untersuchten Salze sind folgende:

$$
\mathrm{AsO}_{4} \mathrm{CaH} \text {. }
$$

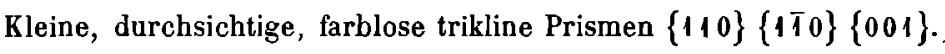

$$
\begin{aligned}
& (110):(1 \bar{T} 0)=56^{0} 58^{\prime} \\
& \text { (110):(001) 10257 } \\
& (1 \overline{1} 0):(001) \quad 828
\end{aligned}
$$

Doppelbr. - . Auslöschungsschiefe auf $(110)+18^{0}$, auf $(1 \overline{1} 0)+38^{0}$; durch (110) und (1 $1 \overline{1} 0)$ je ein Axenbild sichtbar.

$$
\mathrm{AsO}_{4} \mathrm{CaH} . \mathrm{H}_{2} \mathrm{O} \text { (Haidingerit). }
$$

Aus Calciumchlorid- oder Acetatlösung und Arsensäure beim Erhilzen auf $70^{\circ}-100^{\circ}$ (bei $150^{\circ}-200^{\circ}$ bildet sich das vor. Salz). Dünne rectanguläre Täfelchen nach $\left\{\begin{array}{lll}0 & 1 & 0\end{array}\right\}$, deren Spaltbarkeit und optische Eigenschaften mit denen des Haidingerit übereinstimmen.

$$
\mathrm{AsO}_{4} \mathrm{SrH} \text {. }
$$

Kleine, farblose Krystalle derselben Combination, tafelförmig nach $\{110\}$, und mit denselben ebenen Winkeln, wie das $\mathrm{Ca}$-Salz. Doppelbr. - ; Auslöschungsschiefe auf $(110) 31^{\circ}$; durch (110) ein Axenbild sichtbar, Axenebene nahe $\perp\left[\begin{array}{lll}0 & 01\end{array}\right]$.

$$
\mathrm{AsO}_{4} \mathrm{SrH} . \mathrm{H}_{2} \mathrm{O} \text {. }
$$

Die Krystalle bilden sich neben denen von $\mathrm{AsO}_{4} \mathrm{SrNa} .9 \mathrm{H}_{2} \mathrm{O}$, wenn man die Lösungen von Strontiumnitrat und Dinatriumarseniat ineinander diffundiren lässt. Farblose, rhombische Prismen $\{110\}$ mit $\left\{\begin{array}{lll}1 & 0 & 0\end{array}\right.$ und einer gerundeten Bipyramide als Endigung. $(110):(100)=44^{0} 5^{\prime}$. Spaltb. $\{100\}$ vollkommen. Ebene der opt. Axen (010), Axe $a$ zweite Mittellinie (wie in dem wahrscheinlich isomorphen Haidingerit).

$$
\mathrm{AsO}_{4} \mathrm{BaH} \text {. }
$$

Bis $\frac{1}{2} \mathrm{~cm}$ grosse trikline Comb.: $\{001\}\{1 \bar{T} 0\}\{110\}\{010\}$. Beob.: (110): $(1 \bar{T} 0)=56^{0} 30^{\prime},(1 \bar{T} 0):(001)=85^{0} 4^{\prime},(110):(001)=103^{0} 23^{\prime}{ }^{\prime},(110):(010)$ $=56^{0} 16^{\prime},(1 \overline{1} 0):\left(00^{\top} 0\right)=67^{0} 11^{\prime}$.

$$
\mathrm{AsO}_{4} \mathrm{BaH} . \mathrm{H}_{2} \mathrm{O} \text {. }
$$

Krystalle aus essigsaurer Lösung in der Kälte. Rhombisch.

$$
\left.a: b: c=0,9380: 1: 0,5642^{1}\right) .
$$

Comb.: $\{100\}\{110\}\{121\}$.

1) Der Verf. giebt an 0,573 . 
Spaltb. $\{100\}$.

Beobachtet :

$$
\begin{aligned}
& (110):(100)={ }^{*} 43^{0} 10^{\prime} \\
& (121):(100) \quad * 68 \quad 15 \\
& (121):(110) \quad 4132
\end{aligned}
$$

Berechnet :

$$
\text { - }
$$

Ebene der opt. Axen $\left\{\begin{array}{lll}0 & 10\end{array}\right\}, c$ erste Mittellinie, $2 E=34^{\circ}$ ca., schwache Dispersion $\varrho<v$.

Die Krystalle sind isomorph mit der Strontium- und wahrscheinlich auch mit der Calciumverbindung.

Bei der Darstellung durch Wechselzersetzung von Baryumnitrat und Natriumarseniat erhält man grosse, aber sehr dünne, rectanguläre Tafeln nach $\{100\}$ mit Abstumpfungen unter $45^{0}[?$ d. Ref.]

$$
\mathrm{AsO}_{4} \mathrm{ZnH} . \mathrm{H}_{2} \mathrm{O} \text {. }
$$

Dünne monokline Täfelchen $\{010\}$, begrenzt von $\{110\},\{011\}$ und $\{101\}$. U. d. M. beob.: $(100):(001)=87^{0} 25^{\prime},(101):(100)=40^{\circ} 25^{\prime}$. Zuweilen Zwillinge nach (100). Doppelbr. + , schwacb; Ebene der opt. Axen $\perp(010)$ bildet mit $c 23^{0} \mathrm{im}$ stumpfen Winkel $\beta$; Axe $b$ zweite Mittellinie.

$$
\left[\mathrm{AsO}_{4}\right]_{4} \mathrm{Zn}_{5} \mathrm{H}_{2} \text {. }
$$

Erhalten im geschlossenen Rohre bei $200^{\circ}$. Dünne rhomboidische Täfelchen, deren Winkel $130^{\circ}$ und $50^{\circ}$, mit einer schiefen Abstumpfung; nach einer Seite Spaltbarkeit; Auslöschungsschiefe gegen diese 14${ }^{\circ}$. Eine opt. Axe nahe 1 zur Tafelfläche, daher wahrscheinlich triklin.

Ausserdem existirt noch ein Salz von der empirischen Formel $\mathrm{As}_{2} \mathrm{O}_{8} \mathrm{Zn}_{3} .8 \mathrm{H}_{2} \mathrm{O}$, welches aber ein Mol. $\mathrm{H}_{2} \mathrm{O}$ schwerer abgiebt; Nadeln mit einer Auslöschungsschiefe von $30^{\circ}-35^{\circ}$.

$$
\mathrm{AsO}_{4} \mathrm{CuH} . \mathrm{H}_{2} \mathrm{O} \text {. }
$$

Hellgrüne monokline Täfelchen nach $\left\{\begin{array}{lll}0 & 10\end{array}\right\}$, begrenzt von $\left\{\begin{array}{lll}1 & 0 & 0\end{array}\right\},\left\{\begin{array}{lll}0 & 0 & 1\end{array}\right\}$,

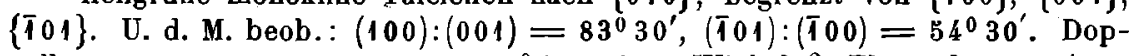
pelbr. $\gamma-\alpha=0,0752, c: c=13^{0}$ im spitzen Winkel $\beta$; Ebene der opt. Axen $\left(\begin{array}{llll}0 & 1 & 0\end{array}\right)$.

$$
\left[\mathrm{AsO}_{4}\right]_{2} \mathrm{Cu}_{3} \text {. }
$$

Dargestellt von Coloriano (Thèse d. l. Fac. d. sc. d. Paris s. l. arséniates crist. 1886). Monokline Prismen oder Täfelchen nach $\{010\}$. Mikroskopische Messungen: $(100):(001)=78^{0} 24^{\prime},\left(T_{01}\right):(001)=37^{0} 36^{\prime}$. Ebene der opt. Axen $(010), a: c=23^{\circ}$ im spitzen Winkel $\beta$; durch $(100)$ ein Axenbild, darnach $\mathfrak{a}$ erste Mittellinie. Pleochroïsmus: $\mathfrak{a}$ grün, $\mathfrak{c}$ blau.

$$
\mathrm{AsO}_{4} \mathrm{Ag}_{3} \text {. }
$$

Rubinrothe, einfachbrechende Krystalle $\{110\}$, zuweilen nach einer trigonalen Axe prismatisch, oder Tetraëder (vergl. diese Zeitschr. 13, 645).

$$
\mathrm{AsO}_{3} \mathrm{Hg} \text {. }
$$

Hexagonale Täfelchen $\{0004\}\{10 T 1\} . \quad a: c=1: 1,5096 . \quad(10 \bar{T} 1):(10 \bar{T} T)$ $=59^{0} 41^{\prime}$. Doppelbr. stark + .

$$
\mathrm{AsO}_{4} \mathrm{Hg}_{3} \text {. }
$$

Rhombisch. Mikroskopische Tafeln $\{001\}$, begrenzt von $\{011\}$ und $\{110\}$;

1) Vom Ref. berechnet; der beobachtete Werth ist wahrscheinlich durch Druckfehler entstellt. 
$(110):(1 \bar{T} 0)=62^{\circ}$. Doppelbr. stark, -; Ebene der opt. Axen (100), c erste Mittellinie, Axenwinkel klein mit sehr grosser Dispersion. Pleochroïsmus: $\mathfrak{a}$ hellbraun, $\mathfrak{b}$ braun, c bräunlichgrün.

$$
\left[\mathrm{AsO}_{4}\right]_{2} \mathrm{Hg}_{3} \text {. }
$$

Monokline gelbe, dünne Prismen $\{110\}$ mit $\{100\},\{010\},\{111\}$ und einer nicht messbaren Form $\{\bar{h} k l\}$.

$$
\begin{aligned}
& (110):(1 \overline{1} 0)=79^{\circ} 59^{\prime} \\
& (111):(1 \overline{1}) \quad 4718
\end{aligned}
$$

Doppelbr. +; Axenebene (010); Auslöschungsschiefe auf $\{110\} 14^{0}-15^{0}$, auf $\left\{\begin{array}{lll}0 & 0\end{array} \quad 18^{\circ} \mathrm{im}\right.$ stumpfen Winkel $\beta$, entsprechend der ersten Mittellinie c; durch $\left\{\begin{array}{lll}1 & 0 & 0\end{array}\right\}$ ein Axenbild sichtbar.

$$
\mathrm{As}_{2} \mathrm{O}_{7}[\mathrm{Ni.OH}]_{2} \mathrm{H}_{2} \text {. }
$$

Krystall. im geschlossenen Rohre über $180^{\circ}$ und giebt erst über $180^{\circ}$ erhitzt Wasser ab. Monokline Täfelchen $\{010\}$, begrenzt von $\{001\}$ und $\left\{\begin{array}{lll}1 & 10 & 0\end{array} ;\right.$ u. d. M. beob.: $(001):(100)=45^{0} 30^{\prime}$. Erste Mittellinie der opt. Axen $\perp(010)$, zweite Mittellinie (c) bildet $28^{0}$ mit $c$ im spitzen Winkel $\beta$.

Von Kobalt wurde ein Salz $\mathrm{As}_{2} \mathrm{O}_{9} \mathrm{CO}_{2} \mathrm{H}_{4}$ erhalten in nicht messbaren Krystallen, welche nach ihren optischen Eigenschaften monoklin sind.

$$
\mathrm{AsO}_{4} \mathrm{MnH} . \mathrm{H}_{2} \mathrm{O} \text {. }
$$

Rosarothe monokline Täfelchen $\left\{\begin{array}{lll}0 & 1 & 0\end{array}\right\}$ mit $\left\{\begin{array}{lll}1 & 0 & 0\end{array}\right\},\left\{\begin{array}{lll}1 & 0 & 1\end{array}\right\},\left\{\begin{array}{ll}1 & 01\end{array}\right\},\left\{\begin{array}{lll}0 & 01\end{array}\right\}$. U. d. M. beobachtet:

$$
\begin{aligned}
& (100):(001)=83^{0} 30^{\circ} \\
& (101):(100) \quad 4548 \\
& (\overline{1} 01):(101) \quad 80 \quad 6
\end{aligned}
$$

Spaltbarkeit nach $\left\{\begin{array}{lll}1 & 0 & 0\end{array}\right\}$.

Ebene der optischen Axen $(010) ; c$ bildet mit $c 20^{\circ}$ im spitzen Winkel $\beta$; $\gamma-\alpha=0,087$.

Isomorph mit der Kupferverbindung (S. 206).

$$
\mathrm{AsO}_{4} \mathrm{Al} \text {. }
$$

Oktaëderähnliche, nicht messbare Kryställchen, nach den optischen Eigenschaften monoklin (Axenebene $\perp\left(\begin{array}{lll}0 & 10)\end{array}\right)$, erste Mittellinie in $(010)$ ).

$$
\mathrm{AsO}_{4} \mathrm{PbH} \text {. }
$$

Talkähnliche weisse Blättchen; monoklin $\left\{\begin{array}{lll}0 & 0 & 0\end{array}\right\}$ mit den Randflächen $\left\{\begin{array}{lll}1 & 1 & 0\end{array}\right\}$ und $\{011\}$. U. d. M. beob.: $(100):(001)=83^{0} 24^{\prime}$. Doppelbr. schwach; Axenebene (010), Auslöschungsschiefe (a) $21^{0}-22^{\prime}$ im spitzen Winkel $\beta$.

$$
\mathrm{AsO}_{4}\left(\mathrm{UO}_{2}\right)_{2} .5 \mathrm{H}_{2} \mathrm{O} \text {. }
$$

Citrongelbe quadratische Blättchen, einaxig negativ.

$$
\left[\mathrm{SbO}_{4}\right]_{2} \mathrm{MgH}_{4} \cdot 1 \mathrm{OH}_{2} \mathrm{O} \text {. }
$$

Mikroskopische pseudohexagonale Prismen, deren Basis $\|$ den Seiten in sechs Sectoren getheilt ist, deren Schwingungsrichtungen $\perp$ zu den Seilen sind; die Prismenflächen zeigen Sanduhrstructur mit entgegengesetzter Doppelbrechung der den Prismen- und den Basisflächen anliegenden Theile.

Das analoge $\mathrm{Ni}$-Salz bildet blaugrüne hexagonale, optisch einaxige Täfelchen mit ziemlich starker positiver Doppelbrechung. Das Co-Salz (roth) ebensolche, welche aber Andeutung von optischer Zweiaxigkeit zeigen.

Ref. : P. Groth. 
37. F. Chaves (in Sevilla): Ueber die Finschlïsse in Quarzkrystallen aus Andalusien (Anales de la Soc. Esp. de Hist. Nat. 1896, 25, 243 ).

Verf. untersuchte hauptsächlich Exemplare aus Morón, Osuna und Puerto real. Die Krystalle finden sich zerstreut im Nummulitenkalk, in Thonen und bunten Mergeln; besonders häufig aber in Gypsschichten von rother Färbung, die bei den oben genannten häufig auftreten. Sie zeigen nur die gewöhnliche Combination, Prisma und Pyramide, keine hemiëdrischen Formen. Verf. zieht daraus den sonderbaren Schluss, die Kryslalle dürften keine Circularpolarisation zeigen, und behauptet, dies an sehr genau senkrecht zur c-Axe geschnittenen Platten nachgewiesen zu haben (I).

Die Farbe wechselt sehr. Es wurden gefunden milchweisse, topasfarbene, honigbraune, blutrothe, dunkelbraune und ganz schwarze Exemplare. Meist geht die Färbung beim Erhitzen verloren oder ändert sich doch sehr stark, was beweist, dass dieselbe durch organische Substanzen hervorgebracht war. Verf. vermuthet, dass die Krystalle aus Lösungen entstanden sind, die Humussubstanzen enthielten. Nur in Exemplaren von bestimmter rother Farbe fanden sich Einschlüsse von Eisenoxyd. Einschlüsse von Flüssigkeiten und Gasen wurden häufig beobachtet; dieselben bestehen aus Kohlendioxyd und Kohlenwasserstoffen.

Ref.: W. Muthmann.

38. S. Calderon (in Sevilla): Spanische Plagioklase (Ebenda, Actas 23). - Eine Zusammenstellung bekannler spanischer Fundorte von Natronfeldspäthen.

Mikroklin findet sich in den Dioriten von Asturien, in den Gneissen von Goirriz und von San Come (Galizien).

A lbit. Die Angabe von Naranjo ${ }^{1}$ ) über ein Vorkommen in Hiendelaencina ist wahrscheinlich falsch; nach des Verfs. Untersuchung findet sich dort nur Oligoklas. Dagegen wurde Albit in kleinen Krystallen beim Torre de Calaturco, Almuñecar, gefunden.

Oligoklas. Grosse, bis $1 \mathrm{dcm}$ lange Krystalle finden sich bei Hiendelaencina; dieselben zeigen oft Einschlüsse von Bleiglanz. Bekannt sind auch die Oligoklase von Ciudad de Avila und von San Ildefonso. Bei Coripe, Prov. Sevilla, findet sich ein blaues Vorkommen, $1,5-2 \mathrm{~cm}$ grosse Krystalle, von denen schöne Exemplare in der Sammlung zu Sevilla aufgestellt sind.

An verschiedenen Orten Asturiens sind grünliche, bis $2 \mathrm{dcm}$ grosse Krystalle gefunden worden ${ }^{2}$ ); auch im Nordosten von Almadén ist das Mineral von Naranjo beobachtet worden.

Anorth it von Osann am Cabo di Gata beobachtet; Verf. fand das Mineral am Calvario bei Morón.

$$
\text { Ref.: W. Muth mann. }
$$

1) Elementas de Mineralogia general, Madrid 1862, 470.

2) Barrois, Recherches sur les terrains anciens des Asturies et de la Galice. Lille 1882, $130-145$. 